\title{
A dislocation density-based continuum model of the anisotropic shock response of single crystal $\alpha$-cyclotrimethylene trinitramine.
}

\author{
D.J. Luscher ${ }^{\mathrm{a}}$, F.L. Addessio ${ }^{\mathrm{a}}$, M.J. Cawkwell ${ }^{\mathrm{a}}$, K.J. Ramos ${ }^{\mathrm{b}}$ \\ ${ }^{a}$ Theoretical Division \\ ${ }^{b}$ Explosive Science and Shock Physics Division \\ Los Alamos National Laboratory \\ Los Alamos, NM, 87545, USA
}

\begin{abstract}
We have developed a model for the finite deformation thermomechanical response of $\alpha$-cyclotrimethylene trinitramine (RDX). Our model accounts for nonlinear thermoelastic lattice deformation through a free energy-based equation of state developed by Cawkwell et al. (2016) in combination with temperature and pressure dependent elastic constants, as well as dislocation-mediated plastic slip on a set of slip systems motivated by experimental observation. The kinetics of crystal plasticity are modeled using the Orowan equation relating slip rate to dislocation density and the dislocation velocity developed by Austin and McDowell (2011), which naturally accounts for transition from thermally-activated to dislocation drag limited regimes. Evolution of dislocation density is specified in terms of local ordinary differential equations reflecting dislocation-dislocation interactions.

The paper presents details of the theory and parameterization of the model, followed by discussion of simulations of flyer plate impact experiments. Impact conditions explored within this combined simulation and experimental effort span shock pressures ranging from 1 to 3 GPa for four crystallographic orientations and multiple specimen thicknesses. Simulation results generated using this model are shown to be in strong agreement with velocimetry measurements from the corresponding plate impact experiments. Finally, simulation results are used to motivate conclusions about the nature of dislocation-mediated plasticity in RDX.
\end{abstract}

Keywords: crystal plasticity, dislocations, RDX, shock loading

\section{Introduction}

Explosives can initiate under impacts whose energy, if distributed homogeneously throughout the material, translates to temperature increases that are insufficient to drive the rapid chemistry observed (Bowden and Yoffe, 1948, 1952). Heterogeneous thermomechanical interactions at the meso-scale (i.e. between single crystal and homogenized macroscale) lead to the formation of localized hot spots within the material. Direct numerical simulations of meso-scale responses can contribute to our understanding of the evolution of hot spots if they include the relevant deformation mechanisms that are essential to the nonlinear thermomechanical response of explosive molecular crystals.

Crystal plasticity modeling is a mature field in the context of applications to quasi-static deformation problems. Early crystal plasticity models employed phenomenological expressions for the plastic slip kinetics and hardening relations and did not include an explicit representation of dislocations (e.g. Asaro, 1983; Mathur and Dawson, 1989; Kalidindi et al., 1992; Bronkhorst et al., 1992). More recent contributions have introduced dislocation density as an evolving field variable (e.g. Roters et al., 2000; Prasad et al., 2005; Ma et al., 2006; Lee et al., 2010; Alankar et al., 2011). Most of these works have focused on metallic single crystals typically comprising cubic or hexagonal crystal symmetry.

There are fewer examples of previous work focused on modeling molecular single crystals, where each lattice site contains a molecule rather than an individual atom. Energetic composites typically employ 
explosive molecular crystals bonded together with a polymeric adhesive. Thus, meso-scale modeling of explosives motivates improved dynamical models for the response of energetic molecular crystals.

Winey and Gupta (2006) developed a thermomechanical framework for simulating the shock response of single crystals that is strongly connected with its incremental update scheme. For example, their nonlinear thermoelasticity is not based on a governing potential, which could be parameterized from measurement or finer scale atomistic simulations, and instead uses an incremental update approach based on a set of corotating elastic moduli and constant Grüneisen tensor. Their approach yielded excellent agreement between measured and simulated velocimetry for impact along the [100] crystallographic direction in single crystal LiF over a range of specimen thicknesses. They extended their dislocation-based plasticity model for application to shock response of the energetic molecular crystal PETN by including a crystallographic shear cracking model. They further extended the incremental linear elasticity to account for nonlinearity by using thirdorder elastic constants and showed that the nonlinear extension is critical for correctly capturing shock wave profiles. Their dislocation theory defines the velocity of dislocations within the drag-limited regime although it does not restrict dislocation velocity to be less than the shear wave speed. As a consequence, the model may not perform as well for situations in which the material is dislocation starved (i.e. high dislocation velocity) nor at lower dislocation velocities when thermally activated motion past dislocation barriers is the dominant mechanism.

Barton et al. (2009) developed a dislocation-based model for the shock response of the energetic molecular single crystal HMX and calibrated it against interface velocimetry results from four impacts spanning two crystal orientations and two specimen thicknesses. Their model accounts for the phenomenology of both thermally activated and drag-limited dislocation motion. Barton et al. (2009) and Austin et al. (2014, 2015) have applied extensions of this model to predicting the evolving temperature field and coupled thermochemical reactions in the vicinity of a collapsing pore in single crystal HMX.

Clayton and Becker (2012) have developed a single crystal model for the quasi-static deformation of $\alpha$-RDX which they applied to simulating thermomechanical responses during nanoindentation experiments. They included six slip systems identified from previous indentation experiments (Gallagher et al., 1992; Ramos et al., 2009). The crystallographic slip rate used in their model is based on a power law dependence of the resolved shear stress. Simulated results of the indenter force versus applied displacement demonstrate consistent trends with measurements, although they exhibit discrepancies of approximately $50 \%$ with the experimental data points in some cases. They further demonstrated that the simulation results are sensitive to elastic moduli and assumed slip system strength parameters over credible ranges in these parameter values.

De et al. (2014) developed a model for the anisotropic single crystal response of RDX to shock loading conditions, which they calibrated and compared to interface velocimetry data from Hooks et al. (2006) and Cawkwell et al. (2010) for shock impacts on the (111) and (210) crystallographic planes. Competing mechanisms of thermally activated dislocation motion and phonon drag at high velocity are used as motivation for a phenomenological viscoplastic flow stress applied at a slip system level in their model; however, they do not explicitly account for the evolving dislocation density field, nor the corresponding velocities of mobile dislocations. Furthermore, the model of De et al. (2014) only includes three distinct slip systems. Thus while they were able to capture aspects of the measured velocimetry data for two impact orientations, that model is unable to reproduce the elastic-plastic two-wave structure observed for impact on (100) orientations as reported in Hooks et al. (2006, 2011). Furthermore, De et al. (2014) compare their simulation results with experimental data reported by Hooks et al. (2006) that was superseded by data published in an erratum by Hooks et al. (2011).

Josyula et al. (2016) applied a variation on the model developed by De et al. (2014) to simulate quasistatic compression. Whereas Josyula et al. (2016) emphasize the importance of representing dislocation behavior, they omit some of the dislocation-inspired phenomenology of De et al. (2014) instead employing a power-law viscoplastic slip rate. While the modified model includes four additional slip systems not included by De et al. (2014), the results exhibit purely elastic response for compression on (100) planes because the considered slip systems have zero Schmid factor for that orientation.

Advances in experimental diagnostics under development within the shock physics community, such as in situ X-ray diffraction and imaging, will provide a better understanding of the mechanisms that accommodate 
deformation imposed through impact loading (Ramos et al., 2014b). In order to simulate, and ultimately predict, the types of data that will be measured by such diagnostics, models must have a consistent physical basis that explicitly accounts for the evolution of crystal defects.

A new model for the anisotropic, dynamic response of $\alpha$-RDX is developed here. The model adopts a multiplicative decomposition of deformation consistent with classical finite deformation crystal plasticity models. Nonlinear thermoelasticity is handled in a thermodynamically consistent framework. The thermoelastic deformation is decoupled in order to carefully account for contributions to free energy from purely volumetric deformation based on the equations of state developed by Cawkwell et al. (2016) and parts representing the coupling between dilatation and isochoric deformation of the lattice. The evolution of plastic deformation is based on crystallographic slip, in which the slip rates are related to dislocation activity through the Orowan equation. The velocity of mobile dislocations reflects the natural temporal competition between thermally activated motion past obstacles impeding dislocation motion and phonon drag-limited dislocation glide using an expression for dislocation velocity developed by Austin and McDowell (2011). We fit the model to one plate impact experiment and compare its predictions to multiple impacts on other planes at different shock pressures and specimen thicknesses collectively representing 28 other plate impact experiments.

There are other available deformation mechanisms which can compete with crystallographic slip in RDX. For example, under certain loading conditions and deformation rates, RDX is known to cleave along weak planes enabling subsequent inter-particle slip (Wiegand et al., 1991; Elban et al., 1998; Sharma et al., 2001). Also, at sufficiently low temperatures, RDX transforms from the $\alpha$ to $\gamma$ polymorph at shock pressures of approximately $3.8 \mathrm{GPa}$ (Cawkwell et al., 2016). At higher temperatures, transformation from $\alpha$ to $\epsilon$ or direct melting to liquid phase can occur. In this work, we do not account for these other deformation mechanisms. Addessio et al. (2016), Barton et al. (2009), and Winey and Gupta (2010) address solid-solid phase transformation in RDX, solid-liquid melting in HMX, and shear cracking in PETN, respectively. As discussed in Section 4, the loading conditions examined in this paper are clearly below the known shock pressures and temperatures required for phase transformation including melt.

The remainder of this paper is organized as follows. The constitutive framework is presented in Section 2, which is further divided into parts on kinematics 2.1, thermodynamics 2.2 , and thermoelasticity including the equations-of-state 2.3. The dislocation density-based crystal plasticity theory is presented in Section 3 along with heuristic arguments drawn from previous experiments to posit seven new slip systems for $\alpha$-RDX. Section 4 briefly summarizes previous experiments and discusses model parameterization and simulation results in comparison with measurements from experiment. Finally, a brief summary closes the paper in Section 5.

\section{Constitutive Framework}

\subsection{Kinematics}

The total gradient of the deformation field $\mathbf{F}=\mathbf{x} \bar{\nabla}$ is decomposed multiplicatively by

$$
\mathbf{F}=J_{\mathrm{e}}^{1 / 3} \hat{\mathbf{F}}_{\mathrm{e}} \mathbf{F}_{\mathrm{p}}
$$

where $\mathbf{x}$ are the deformed positions of material points, $\nabla$ is the gradient operator with respect to reference configuration positions, the determinant of deformation gradient is $J_{\mathrm{e}}=\operatorname{det} \mathbf{F}_{\mathrm{e}}, \widehat{\mathbf{F}}_{\mathrm{e}}=J_{\mathrm{e}}^{-1 / 3} \mathbf{F}_{\mathrm{e}}$ is the isochoric part of the elastic deformation, and $\mathbf{F}_{\mathrm{p}}$ is the traditional plastic part of the deformation gradient associated with the accumulation of crystallographic slip. This relationship implies that the traditional elastic deformation gradient, $\mathbf{F}_{\mathrm{e}}$, is decomposed into two separate maps, a volumetric expansion followed by an isochoric deformation, i.e.,

$$
\mathbf{F}_{\mathrm{e}}=\widehat{\mathbf{F}}_{\mathrm{e}} \overline{\mathbf{F}}_{\mathrm{e}}
$$

where the volumetric expansion is $\overline{\mathbf{F}}_{\mathrm{e}}=J_{\mathrm{e}}^{1 / 3} \mathbf{I}$. The conventional elastic Green-Lagrange strain is $\mathbf{E}_{\mathrm{e}}=$ $\frac{1}{2}\left(\mathbf{F}_{\mathrm{e}}^{T} \mathbf{F}_{\mathrm{e}}-\mathbf{I}\right)$. Analogously, we construct isochoric and volumetric measures of Green-Lagrange strain as $\widehat{\mathbf{E}}_{\mathrm{e}}=\frac{1}{2}\left(\widehat{\mathbf{F}}_{\mathrm{e}}^{T} \widehat{\mathbf{F}}_{\mathrm{e}}-\mathbf{I}\right)$ and $\overline{\mathbf{E}}_{\mathrm{e}}=\frac{1}{2}\left(J_{\mathrm{e}}^{2 / 3}-1\right) \mathbf{I}$, respectively. 
Like the total elastic Green-Lagrange strain, the dilatational Green-Lagrange strain is defined within the reference configuration. On the other hand, the isochoric Green-Lagrange strain is defined within a dilatationally expanded configuration, thus we define

$$
\widehat{\mathbf{E}}_{\mathrm{e}}=\bar{\phi}^{*}\left(\widehat{\mathbf{E}}_{\mathrm{e}}\right)=\overline{\mathbf{F}}_{\mathrm{e}}^{T} \widehat{\mathbf{E}}_{\mathrm{e}} \overline{\mathbf{F}}_{\mathrm{e}}
$$

which represents the volumetric pull-back of $\widehat{\mathbf{E}}_{\mathrm{e}}$ in order to facilitate the additive decomposition

$$
\mathbf{E}_{\mathrm{e}}=\overline{\mathbf{E}}_{\mathrm{e}}+\widehat{\widehat{\mathbf{E}}}_{\mathrm{e}}
$$

In this manner, $\widehat{\mathbf{E}}_{\mathrm{e}}$ is that part of $\mathbf{E}_{\mathrm{e}}$ attributed to the isochoric mapping of $\widehat{\mathbf{F}}_{\mathrm{e}}$, i.e., under purely dilatational deformation $\widehat{\mathbf{E}}_{\mathrm{e}}=\mathbf{0}$. Likewise, $\overline{\mathbf{E}}_{\mathrm{e}}$ is that part of $\mathbf{E}_{\mathrm{e}}$ attributed solely to the dilatation of material points by the mapping $\overline{\mathbf{F}}_{\mathrm{e}}=J_{\mathrm{e}}^{1 / 3} \mathbf{I}$; under any purely isochoric deformation, $J_{\mathrm{e}}=1$ and $\overline{\mathbf{E}}_{\mathrm{e}}=\mathbf{0}$. Note that $\overline{\mathbf{E}}_{\mathrm{e}}$ is purely volumetric and does not depend upon deviatoric deformation for any deformation mapping $\mathbf{F}_{\mathrm{e}}$. Correspondingly, $\widehat{\mathbf{E}}_{\mathrm{e}}$ is purely isochoric and does not depend upon $J_{\mathrm{e}}$ for any deformation mapping $\mathbf{F}_{\mathrm{e}}$. However, while $\widehat{\mathbf{E}}_{\mathrm{e}}$ reflects the deviatoric component of deformation, it is scaled by the dilatational response (i.e. $J_{\mathrm{e}}$ ) leading to a kinematic coupling.

The specific volume in the current configuration is defined as $V=\frac{1}{\rho}=\operatorname{det} \mathbf{F} / \rho_{0}$. Restricting our focus here to isochoric plasticity, this is equivalent to $V=J_{e} / \rho_{0}$, although in more general cases including inelastic volumetric deformation, e.g. pore growth, a more careful distinction must be made between the specific volume of the solid material phase and that of the effective homogenized aggregate.

The velocity gradient, $\mathbf{L}=\mathbf{F F}^{-1}$, is pulled into the intermediate configuration where it decomposes additively into elastic and inelastic parts, i.e.,

$$
\widetilde{\mathbf{L}}=\widetilde{\mathbf{L}}_{\mathrm{e}}+\widetilde{\mathbf{L}}_{\mathrm{p}}, \quad \widetilde{\mathbf{L}}_{\mathrm{e}}=\mathbf{F}_{\mathrm{e}}^{-1} \dot{\mathbf{F}}_{\mathrm{e}}, \quad \widetilde{\mathbf{L}}_{\mathrm{p}}=\dot{\mathbf{F}}_{\mathrm{p}} \mathbf{F}_{\mathrm{p}}^{-1}
$$

The symmetric rate of deformation tensor in the intermediate configuration is likewise obtained by pull back of $\mathbf{D}=\operatorname{sym}(\mathbf{L})$ via $\mathbf{F}_{\mathrm{e}}$ resulting in

$$
\widetilde{\mathbf{D}}=\widetilde{\mathbf{D}}_{\mathrm{e}}+\widetilde{\mathbf{D}}_{\mathrm{p}}, \quad \widetilde{\mathbf{D}}_{\mathrm{e}}=\frac{1}{2}\left(\mathbf{C}_{\mathrm{e}} \widetilde{\mathbf{L}}_{\mathrm{e}}+\widetilde{\mathbf{L}}_{\mathrm{e}}^{T} \mathbf{C}_{\mathrm{e}}\right), \quad \text { and } \quad \widetilde{\mathbf{D}}_{\mathrm{p}}=\frac{1}{2}\left(\mathbf{C}_{\mathrm{e}} \widetilde{\mathbf{L}}_{\mathrm{p}}+\widetilde{\mathbf{L}}_{\mathrm{p}}^{T} \mathbf{C}_{\mathrm{e}}\right)
$$

where $\mathbf{C}_{\mathrm{e}}=\mathbf{F}_{\mathrm{e}}^{T} \mathbf{F}_{\mathrm{e}}$ is the elastic Cauchy deformation tensor.

\subsection{Thermodynamics}

The nonlinear thermoelastic behavior of the material is defined via an expression for the Helmholtz free energy as a state function of the elastic Green-Lagrange strain, $\mathbf{E}_{e}$, temperature, $T$, and a set of dislocation densities, $\varrho^{\alpha} \forall \alpha \in[1, N]$ on $N$ distinct crystallographic slip systems, i.e.,

$$
\psi=\hat{\psi}\left(\mathbf{E}_{e}, T, \varrho^{\alpha}\right)
$$

Following standard thermodynamic arguments within the context of internal state variable theory leads to the usual state relations,

$$
\mathbf{S}=\tilde{\rho} \frac{\partial \hat{\psi}}{\partial \mathbf{E}_{\mathrm{e}}} \quad s=-\frac{\partial \hat{\psi}}{\partial T} \quad f^{\alpha}=\tilde{\rho} \frac{\partial \hat{\psi}}{\partial \varrho^{\alpha}}
$$

where $\mathbf{S}$ is the quasi-conservative second Piola-Kirchhoff stress with respect to the intermediate configuration, $s$ is the entropy, and $f^{\alpha}$ is a thermodynamic force related to the stored energy of dislocations. Consistent with thermodynamic arguments of Ziegler (1983), we decompose the total stress, $\mathbf{S}_{\text {tot }}$ into quasi-conservative and dissipative parts, i.e., $\mathbf{S}_{\text {tot }}=\mathbf{S}+\mathbf{S}_{\text {diss }}$. The dissipative contribution to total stress, $\mathbf{S}_{\text {diss }}$, reflects the departure from thermodynamic equilibrium and conservation of momentum is imposed upon the total stress field, $\mathbf{S}_{\text {tot }}$ (Luscher et al., 2013). 
Neglecting heat flux, the rate of temperature change can be expressed as

$$
\tilde{\rho} C_{V} \dot{T}=\tilde{\Phi}_{\text {mec }}+\tilde{\rho} T\left(\frac{\partial^{2} \hat{\psi}}{\partial \mathbf{E}_{\mathrm{e}} \partial T}: \widetilde{\mathbf{D}}_{\mathrm{e}}+\sum_{\alpha} \frac{\partial^{2} \hat{\psi}}{\partial \varrho^{\alpha} \partial T} \dot{\varrho}^{\alpha}\right)
$$

where $\tilde{\Phi}_{\text {mec }}$, is the rate of mechanical dissipation per unit volume (on the intermediate configuration),

$$
\tilde{\Phi}_{\mathrm{mec}}=\mathbf{S}: \widetilde{\mathbf{D}}_{\mathrm{p}}-\sum_{\alpha} f^{\alpha} \dot{\varrho}^{\alpha}+\mathbf{S}_{\mathrm{diss}}: \widetilde{\mathbf{D}}
$$

and the heat capacity at constant volume is defined as $C_{V}=T \frac{\partial s}{\partial T}$. The first term on the right hand side of Eq. (10) reflects contributions from the plastic power, the second term reflects the rate energy is stored to evolve microstructure, e.g. the change in trapped lattice strain energy, and the last term is dissipation caused by a non-equilibrium transition between two (near) equilibrium states. Equations 8 and 9 are typical results of the thermodynamical analysis of irreversible processes in the context of finite deformation plasticity employing a multiplicative decomposition of the deformation gradient and casting the constitutive equations in the intermediate configuration (Scheidler and Wright, 2001; Clayton, 2005).

\subsection{Thermoelasticity}

For crystals exhibiting less than cubic symmetry, the free energy cannot be additively decomposed into purely volumetric and deviatoric parts. Instead, we decompose into a purely volumetric part and a part that represents the thermoelastic coupling between isochoric and volumetric deformation. The Helmholtz free energy corresponding to linear thermoelasticity can be extended to finite deformation according to

$$
\tilde{\rho} \hat{\psi}_{\mathrm{lin}}=\frac{1}{2} \mathbf{E}_{\mathrm{e}}: \mathbb{C}: \mathbf{E}_{\mathrm{e}}-\mathbf{E}_{\mathrm{e}}: \mathbb{C}: \boldsymbol{\alpha}-\frac{C \mathrm{v}}{2 T_{\mathrm{ref}}}\left(T-T_{\mathrm{ref}}\right)^{2}+\tilde{\rho} s_{\mathrm{ref}}\left(T-T_{\mathrm{ref}}\right)+\tilde{\rho} \psi_{\mathrm{ref}}
$$

where $\mathbb{C}$ is the fourth-rank elastic stiffness tensor, $\boldsymbol{\alpha}$ is a second-rank tensor of stress-free thermal expansion (which can be generalized to include a nonlinear dependence on $T$ ), $T_{\text {ref }}$ is a reference temperature, $s_{\text {ref }}$ and $\psi_{\text {ref }}$ are the entropy and Helmholtz free energy under reference conditions $\left(T=T_{\text {ref }}, \mathbf{E}_{\mathrm{e}}=\mathbf{0}\right)$, respectively. We assume that the thermal strains, $\boldsymbol{\alpha}$, have a small deviatoric component permitting the decomposition $\boldsymbol{\alpha}=\hat{\boldsymbol{\alpha}}+\frac{1}{3} \alpha_{v} \mathbf{I}$, where $\hat{\boldsymbol{\alpha}}$ and $\alpha_{v}$ are the deviatoric and volumetric parts of the thermal strain tensor, respectively. Note, our notation here is somewhat nonstandard in that $\boldsymbol{\alpha}$ is the stress-free thermal strain rather than linear coefficients of thermal expansion, thus $\mathbb{C}: \boldsymbol{\alpha}$ in our Eq. 11 is directly analogous to $\boldsymbol{\beta} \Delta T$ in Eq 5.85 of Clayton (2011). We use this nomenclature in order to readily permit a nonlinear relationship between thermal strain and temperature as measured by Cady (1972).

Substitution of the decomposition from Eq. 4 into Eq. 11 enables a decomposition of the free energy of quasi-linear thermoelasticity

$$
\hat{\psi}_{\text {lin }}=\hat{\psi}_{\text {vol }}(V, T)+\hat{\psi}_{\mathrm{cpl}}\left(\widehat{\mathbf{E}}_{\mathrm{e}}, V, T\right)
$$

into a term that only depends upon specific volume, $\hat{\psi}_{\text {vol }}$, and a term, $\hat{\psi}_{\mathrm{cpl}}$, that depends on the volumetric and isochoric parts of deformation in a coupled manner, where

$$
\tilde{\rho} \hat{\psi}_{\mathrm{vol}}(V, T)=\frac{1}{2} K \delta^{2}-K \delta \bar{\alpha}-\frac{C_{V}}{2 T_{\mathrm{ref}}}\left(T-T_{\mathrm{ref}}\right)^{2}+\tilde{\rho} s_{\mathrm{ref}}\left(T-T_{\mathrm{ref}}\right)+\tilde{\rho} \psi_{\mathrm{ref}}
$$

and

$$
\tilde{\rho} \hat{\psi}_{\mathrm{cpl}}=\frac{1}{2} \widehat{\mathbf{E}}_{\mathrm{e}}: \mathbb{C}: \widehat{\mathbf{E}}_{\mathrm{e}}+\widehat{\mathbf{E}}_{\mathrm{e}}: \mathbb{C}: \overline{\mathbf{E}}_{\mathrm{e}}-\left[\widehat{\mathbf{E}}_{\mathrm{e}}: \mathbb{C}: \hat{\boldsymbol{\alpha}}+\widehat{\mathbf{E}}_{\mathrm{e}}: \mathbf{G} \alpha_{v}+\overline{\mathbf{E}}_{\mathrm{e}}: \mathbb{C}: \hat{\boldsymbol{\alpha}}\right]
$$

where we define $\frac{1}{3} \delta=\frac{1}{2}\left(J_{\mathrm{e}}^{2 / 3}-1\right)$ and $K=\frac{1}{9} \mathbf{I}: \mathbb{C}: \mathbf{I}$. The coupled contribution of deviatoric deformation to free energy can be expressed more simply as

$$
\tilde{\rho} \hat{\psi}_{\mathrm{cpl}}=\frac{1}{2} \mathbf{E}_{\mathrm{e}}: \mathbb{C}: \mathbf{E}_{\mathrm{e}}-\mathbf{E}_{\mathrm{e}}: \mathbb{C}: \boldsymbol{\alpha}-\left[\frac{1}{2} K \delta^{2}-K \delta \alpha_{v}\right]
$$


In order to model the nonlinear thermoelastic response of the material we replace the purely volumetric contributions to Eq. 11 with a parameterized Helmholtz free energy function representing the pressurevolume-temperature equation of state $(\mathrm{EOS})$, i.e., $\hat{\psi}_{\text {vol }}(V, T) \leftarrow \hat{\psi}_{\text {eos }}(V, T)$ such that

$$
\hat{\psi}=\hat{\psi}_{\mathrm{eos}}(V, T)+\hat{\psi}_{\mathrm{cpl}}\left(\widehat{\mathbf{\mathbf { E }}}_{\mathrm{e}}, V, T\right)+\hat{\psi}_{\mathrm{str}}\left(\varrho^{\alpha}, T\right)
$$

where $\hat{\psi}_{\text {str }}$ represents the free energy stored in the lattice due to evolving density and configuration of dislocations. We use the Helmholtz free energy function developed and parameterized for $\alpha$-RDX by Cawkwell et al. (2016) to define the equations of state represented by $\hat{\psi}_{\text {eos }}(V, T)$ in Eq. 16. The second Piola-Kirchhoff stress with respect to the intermediate configuration is obtained from the state relations of Eq. 8 resulting in a decomposition $\mathbf{S}=\mathbf{S}_{\mathrm{eos}}+\mathbf{S}_{\mathrm{cpl}}$. The part of second Piola-Kirchhoff stress associated with the EOS is computed by

$$
\mathbf{S}_{\mathrm{eos}}=\tilde{\rho} \frac{\partial \hat{\psi}_{\mathrm{eos}}}{\partial \mathbf{E}_{\mathrm{e}}}=-J_{\mathrm{e}} P_{\mathrm{eos}} \mathbf{C}_{\mathrm{e}}^{-1}
$$

where the pressure evaluated from the EOS is $P_{\text {eos }}=-\frac{\partial \hat{\psi}_{\text {eos }}}{\partial V}$ and $\mathbf{C}_{\mathrm{e}}=\mathbf{F}_{\mathrm{e}}^{T} \mathbf{F}_{\mathrm{e}}$ is the elastic Cauchy deformation tensor acting as metric on the intermediate configuration.

The part of second Piola-Kirchhoff stress attributed to coupling of isochoric and volumetric deformation is computed from Eq. 15 by

$$
\mathbf{S}_{\mathrm{cpl}}=\tilde{\rho} \frac{\partial \hat{\psi}_{\mathrm{cpl}}}{\partial \mathbf{E}_{\mathrm{e}}}=\mathbb{C}:\left(\mathbf{E}_{\mathrm{e}}-\boldsymbol{\alpha}\right)-K J_{\mathrm{e}}^{2 / 3}\left(\delta-\alpha_{v}\right) \mathbf{C}_{\mathrm{e}}^{-1}+\tilde{\rho} \frac{\partial \hat{\psi}_{\mathrm{cpl}}}{\partial P} \frac{\partial P}{\partial \mathbf{E}_{\mathrm{e}}}
$$

where the last term arises due to the pressure dependence of the elastic constants used to parameterize the Helmholtz free energy, i.e., $\mathbb{C}(P, T)$. The corresponding partial derivative of the free energy with respect to pressure (and with elastic deformation held fixed) is

$$
\tilde{\rho} \frac{\partial \hat{\psi}_{\mathrm{cpl}}}{\partial P}=\mathbf{E}_{\mathrm{e}}: \frac{\partial \mathbb{C}}{\partial P}:\left(\frac{1}{2} \mathbf{E}_{\mathrm{e}}-\boldsymbol{\alpha}\right)-\frac{\partial K}{\partial P}\left[\frac{1}{2} \delta^{2}-\delta \alpha_{v}\right]
$$

The Cauchy stress related to Eq. 18 is obtained by the push forward of $\mathbf{S}_{\mathrm{cpl}}$ expressed as

$$
\boldsymbol{\sigma}_{\mathrm{cpl}}=\boldsymbol{\sigma}_{\mathrm{o}}+P_{\mathrm{cor}} \mathbf{I}+\left(\tilde{\rho} \frac{\partial \hat{\psi}_{\mathrm{cpl}}}{\partial P}\right) J_{\mathrm{e}}^{-1} \mathbf{F}_{\mathrm{e}} \frac{\partial P}{\partial \mathbf{E}_{\mathrm{e}}} \mathbf{F}_{\mathrm{e}}^{T}
$$

where $\boldsymbol{\sigma}_{\mathrm{o}}=J_{\mathrm{e}}^{-1} \mathbf{F}_{\mathrm{e}} \mathbf{S}_{\mathrm{o}} \mathbf{F}_{\mathrm{e}}^{T}$ is the elastic push-forward of the linear thermoelastic second Piola-Kirchhoff stress (i.e., $\left.\mathbf{S}_{\mathrm{o}}=\mathbb{C}:\left(\mathbf{E}_{\mathrm{e}}-\boldsymbol{\alpha}\right)\right)$, and $P_{\text {cor }}=-K J_{\mathrm{e}}^{-1 / 3}\left(\delta-\alpha_{v}\right)$.

The pressure related to the contribution to Cauchy stress in Eq. 20 is

$$
P_{\mathrm{cpl}}=P_{\mathrm{o}}-P_{\mathrm{cor}}-\frac{1}{3}\left(\tilde{\rho} \frac{\partial \hat{\psi}_{\mathrm{cpl}}}{\partial P}\right) J_{\mathrm{e}}^{-1} \mathbf{C}_{\mathrm{e}}: \frac{\partial P}{\partial \mathbf{E}_{\mathrm{e}}}
$$

where $P_{\mathrm{o}}=-\frac{1}{3} J_{\mathrm{e}}^{-1} \mathbf{C}_{\mathrm{e}}: \mathbb{C}:\left(\mathbf{E}_{\mathrm{e}}-\boldsymbol{\alpha}\right)$. Accordingly, the total quasi-conservative pressure is expressed by

$$
P=P_{\text {eos }}+P_{\mathrm{o}}-P_{\text {cor }}-\frac{1}{3}\left(\tilde{\rho} \frac{\partial \hat{\psi}_{\mathrm{cpl}}}{\partial P}\right) J_{\mathrm{e}}^{-1} \mathbf{C}_{\mathrm{e}}: \frac{\partial P}{\partial \mathbf{E}_{\mathrm{e}}}
$$

which is a nonlinear first-order differential equation in deformation $\mathbf{E}_{\mathrm{e}}$. It is outside the scope of this paper to assess the relative accuracy of various approximate solutions to Eq. 22 and instead we neglect the contributions from the pressure derivative of the coupled deviatoric expression for free energy and make the approximation for the quasi-conservative Cauchy stress expressed as

$$
\boldsymbol{\sigma}=\boldsymbol{\sigma}_{\mathrm{o}}-\left(P_{\mathrm{eos}}-P_{\mathrm{cor}}\right) \mathbf{I}
$$




\section{Crystal plasticity}

We assume a field of dislocation density (line length per unit volume) distributed amongst $N_{s}$ slip systems such that $\varrho^{\alpha}$ is the density of dislocations on the $\alpha^{\text {th }}$ slip system. Associated with the $\alpha^{\text {th }}$ slip system are unit vectors normal to the slip plane, $\mathbf{n}_{\mathrm{o}}^{\alpha}$, and contained within that plane and aligned with the direction of slip, $\mathbf{s}_{\mathrm{o}}^{\alpha}$. We further assume that the total dislocation density can be decomposed into mobile $\left(\varrho_{M}^{\alpha}\right)$ and immobile $\left(\varrho_{I}^{\alpha}\right)$ parts, i.e.,

$$
\varrho^{\alpha}=\varrho_{M}^{\alpha}+\varrho_{I}^{\alpha}
$$

In this work we do not track the geometrically necessary component of the dislocation density, nor do we separately quantify densities of dislocations of specified character, i.e. edge, screw, or mixed. The evolution of plastic deformation is specified using an expression of Orowan's equation

$$
\dot{\mathbf{F}}_{\mathrm{p}} \mathbf{F}_{\mathrm{p}}^{-1}=\widetilde{\mathbf{L}}_{\mathrm{p}}=\sum_{\alpha=1}^{N_{s}} b^{\alpha} \varrho_{M}^{\alpha} v^{\alpha} \mathbf{s}_{\mathrm{o}}^{\alpha} \otimes \mathbf{n}_{\mathrm{o}}^{\alpha}
$$

where $b^{\alpha}$ is the magnitude of the Burgers vector and $v^{\alpha}$ the signed scalar velocity of mobile dislocations on slip system $\alpha$.

The dislocation velocity is expressed as a constitutive function, i.e. $v^{\alpha}=\hat{v}\left(\tau^{\alpha}, T, \varrho_{M}^{\beta}, \varrho_{I}^{\beta}\right.$, $)$, where the resolved shear stress on slip system $\alpha$ is $\tau^{\alpha}=\mathbf{S}: \mathbf{s}_{\mathrm{o}}^{\alpha} \otimes \mathbf{n}_{\mathrm{o}}^{\alpha}$ and $\beta=\left[1 . . N_{s}\right]$, in general. Following Lloyd et al. (2014) and Luscher et al. (2016), we use the expression developed by Austin and McDowell (2011) for dislocation velocity, which represents the rate-determined transition between thermal activation, viscous drag, and relativistic effects that limit the maximum velocity of dislocations to the shear wave speed on a particular slip system. This model is based on the average velocity at which a dislocation travels between obstacles computed as

$$
v^{\alpha}=\frac{\bar{L}}{t_{w}+t_{r}} \operatorname{sign}\left(\tau^{\alpha}\right)
$$

where $\bar{L}$ is the mean spacing between obstacles. The time a dislocation spends waiting at a barrier, $t_{w}$, represents the probability of a dislocation overcoming the barrier and depends on both the stress-assisted activation energy and the magnitude of thermal fluctuations, i.e.,

$$
t_{w}=\frac{1}{f_{D}}\left(\exp \left[\frac{\Delta G(\tau)}{k_{B} T}\right]-1\right)
$$

where $f_{D}$ is the attempt frequency, $k_{B}$ is Boltzmann's constant, and $\Delta G$ is the stress-assisted activation energy (cf. Alleman et al., 2014, for example). In this case,

$$
\Delta G^{\alpha}(\tau)=\Delta G_{0}\left\langle 1-\left(\frac{\left|\tau^{\alpha}\right|}{\tau_{r}^{\alpha}}\right)^{q_{1}}\right\rangle^{q_{2}}
$$

where $\langle x\rangle=1 / 2(|x|+x), \tau_{r}^{\alpha}$ is the slip resistance on slip system $\alpha$, the activation energy is defined $\Delta G_{0}=$ $g_{0} \mu b^{3}$, and $g_{0}$ is a dimensionless energy barrier coefficient. The parameters $q_{1}$ and $q_{2}$ affect the shape of the energy barrier consistent with the arguments of Kocks et al. (1975). The slip resistance reflects Taylor hardening attributed to the interaction of the total dislocation field, i.e.,

$$
\tau_{r}^{\alpha}=\tau_{0}+\mu b \sqrt{\sum_{\beta} H_{\alpha \beta} \varrho^{\beta}}
$$

where $\tau_{0}$ is an intrinsic lattice resistance to slip and $H_{\alpha \beta}$ the slip system hardening interaction matrix, which represents the interactions of dislocations on each glide plane with dislocations of all other systems (Ma and Roters, 2004; Devincre et al., 2006; Kubin et al., 2008). Devincre et al. (2006) have used discrete dislocation dynamics simulations to investigate and characterize the modes of interaction between dislocations on a 
primary slip system and various intersecting forest systems in order to quantify $H_{\alpha \beta}$ for face-centered cubic metals. Kubin et al. (2008) discuss that such interaction coefficients are dependent on the dislocation density. Much of the work in this area to date has been focused on metals, for which typical dislocation densities are much higher than for explosive molecular crystals. Kubin et al. suggest that when "necessary and to a reasonably good approximation" the detailed interactions represented by a fully populated interaction matrix can be replaced by an average interaction strength. Without extensive characterization of the orientationdependent stress-strain behavior or finer scale calculations pertinent to RDX, we approximate the hardening interaction matrix as $H_{\alpha \beta}=1 / 4$.

The mean transit time between obstacles for a dislocation, $t_{r}$, is related to the drag-limited mean freerunning velocity of mobile dislocations, i.e., $t_{r}=\bar{L} / v_{r}$. Austin and McDowell (2011) compute the mean free-running velocity by integrating an assumed spatial velocity profile between obstacles to obtain a quasilinear viscous drag relationship, i.e.,

$$
v_{r}^{\alpha}=\frac{\tau^{\alpha} b}{B^{\star}}
$$

where $B^{\star}$ is a viscous drag coefficient that increases without bound as $v_{r}$ approaches the shear wave speed, $c_{s}$, according to

$$
B^{\star}=\frac{B_{0}(T)}{1-\left(v_{r} / c_{s}\right)^{2}}
$$

In this way, the so-called relativistic effect restricts dislocation velocities to not exceed the shear wave speed of the material (Austin and McDowell, 2011).

The evolution rates for mobile and immobile fractions of dislocation density are prescribed according to phenomenology representing local dislocation mechanisms including the possibility of homogeneous nucleation, $\dot{\varrho}_{\text {nuc }}^{\alpha}$, the generation of new dislocations through interactions of mobile dislocations encountering forest dislocations, $\dot{\varrho}_{\text {mult }}^{\alpha}$, the mutual self annihilation of dislocations associated with the same slip system, $\dot{\varrho}_{\text {ann }}^{\alpha}$, and the demobilization of dislocations, $\dot{\varrho}_{\text {trap }}^{\alpha}$, according to

$$
\dot{\varrho}_{M}^{\alpha}=\dot{\varrho}_{\text {nuc }}^{\alpha}+\dot{\varrho}_{\text {mult }}^{\alpha}-\dot{\varrho}_{\text {ann }}^{\alpha}-\dot{\varrho}_{\text {trap }}^{\alpha} \quad \text { and } \quad \dot{\varrho}_{I}^{\alpha}=\dot{\varrho}_{\text {trap }}^{\alpha},
$$

respectively. While homogeneous nucleation of dislocations was considered in the theoretical development, our plasticity model for $\alpha$-RDX does not include such terms, i.e. $\dot{\varrho}_{\text {nuc }}^{\alpha}=0$. Nanoindentation experiments conducted by Ramos et al. (2011) correlate the competition between activation of existing dislocation sources and homogeneous nucleation of new dislocations with intitial defect densities and, ultimately, specimen preparation techniques. Their results support the possibility of homogeneous nucleation of dislocations at shear stresses exceeding $800 \mathrm{MPa}$. Our simulation results (cf. Section 4) suggest that, even with low initial dislocation density, shear stresses remain below this threshold for all cases considered here. While it is well accepted that homogeneous dislocation nucleation readily occurs in many metals under shock loading, the homogeneous nucleation of perfect dislocations has not yet been observed in large scale molecular dynamics simulations of impacts on oriented RDX single crystals (Cawkwell et al., 2008, 2010; Ramos et al., 2010; Wood et al., 2015). Therefore, we hypothesize that dislocation sources are generated and activated dynamically during the experiments.

Relative to many plastically-deformable crystalline materials, the RDX crystals synthesized at the Los Alamos National Laboratory Explosive Crystal Lab contain few dislocations prior to significant deformation. Consequently, the rapid generation of dislocations is critical for accommodation of deformation from shock loading by mechanisms of crystallographic slip. Interactions of dislocations with defects is a typical source of new dislocations and previous nanoindentation experiments have shown that sample surface preparation induces significant sources that effect deformation behavior even in crystals with very low bulk dislocation density from growth (Ramos et al., 2011). Here we consider the interaction of mobile dislocations on one slip plane encountering forest dislocations to co-operate as a Frank-Read source. The proportion of dislocations penetrating the $\alpha^{\text {th }}$ slip system is computed by assuming an equal proportion of edge and screw character dislocations on all other slip systems such that

$$
\varrho_{\text {for }}^{\alpha}=\sum_{\beta} A_{\text {forest }}^{\alpha \beta} \varrho^{\beta}
$$


where

$$
A_{\text {forest }}^{\alpha \beta}=\frac{1}{2}\left(\mathbf{n}^{\alpha} \cdot \mathbf{s}^{\beta}+\mathbf{n}^{\alpha} \cdot \mathbf{q}^{\beta}\right)
$$

and $\mathbf{q}^{\beta}=\mathbf{n}^{\beta} \times \mathbf{s}^{\beta}$ is the dislocation line direction for dislocation segments of edge character. The dislocation evolution rate is prescribed following Ma and Roters (2004); Ma et al. (2006) as

$$
\dot{\varrho}_{\text {mult }}^{\alpha}=C_{\mathrm{M}} \sqrt{\varrho_{\text {for }}^{\alpha}} \varrho_{\mathrm{M}}^{\alpha} v^{\alpha}
$$

According to these expressions, the few initial dislocations are driven to high velocity in order to accommodate plastic slip. However, the initial density of dislocations is insufficient to accommodate arbitrarily large deformation rates, especially when the restriction of drag-limited dislocation velocity is considered. Instead, we assume that cross slip, and double cross slip in particular, is responsible for the rapid generation of new Frank-Read sources. Thus, the unusually high rates of dislocation multiplication coefficient, $C_{M}$, used in our model (cf. Table 5) is consistent with experimental observations of extensive cross slip and/or poorly defined slip planes in RDX (Elban et al., 1989; Ramos et al., 2009). For instance, dislocations with Burgers vectors [100] have been observed to glide on (001), \{011\}, and $\{021\}$ (Halfpenny et al., 1986; Ramos et al., 2009).

Barton et al. (2009) avoid making the distinction between mobile and immobile partitions of the dislocation population. Their decision is reasonable in the context of typically low dislocation densities for molecular crystals, where hardening effects from the immobile part of the dislocation density field can be neglected in many cases. Additionally, with low dislocation content and relatively few defects, it is unlikely that a significant fraction of the population is immobile. However, we include a mechanism for trapping of mobile dislocations as they encounter forest dislocations to enable exploration of the relative effects of this mechanism. In our model the rate of trapping is expressed as

$$
\dot{\varrho}_{\text {trap }}^{\alpha}=C_{\mathrm{T}} \sqrt{\varrho_{\text {for }}^{\alpha}} \varrho_{\mathrm{M}}^{\alpha} v^{\alpha}
$$

where $C_{\mathrm{T}}$ is the trapping coefficient. Annihilation of dislocations interacting within the slip system is especially important in representing a limiting saturation dislocation density. While we do not separately track dislocation character in the manner of, for example, Luscher et al. (2016) or Mayeur et al. (2016), the phenomenology considers dislocations of opposite polarity on the same slip system encountering each other within a capture distance, $d_{\mathrm{a}}$, such that they annihilate each other, i.e.,

$$
\dot{\varrho}_{\mathrm{ann}}^{\alpha}=C_{\mathrm{A}} d_{\mathrm{a}} \varrho^{\alpha} \varrho_{\mathrm{M}}^{\alpha} v^{\alpha}
$$

\subsection{Slip system geometry}

The dislocation slip systems of $\alpha$-RDX have been studied intermittently for over 40 years and yet a complete catalog of its underlying deformation mechanisms is not yet available. The mechanisms of dislocation mediated plasticity in RDX, and molecular crystals in general, are difficult to quantify owing to their brittleness and the challenges associated with applying traditional metallurgical characterization techniques such as transmission electron microscopy to them. Nevertheless, notable progress has been made using X-ray topography and indentation with surface characterization.

While enlightening, these techniques also have inherent limitations. A single technique applicable to RDX has not yet been capable of simultaneously identifying both the Burgers vector and slip plane in a single deformation experiment; therefore, unavoidable ambiguity remains in assigning dislocations with a specific Burgers vector to a specific glide plane. Researchers have thus proposed slip systems that are consistent with experimental observations, but they are not necessarily definitive or unique. For example, etch pit alignment or a slip trace following indentation provides a zone axis contained in the slip plane; whereas, the invisibility condition for diffraction provides the Burgers vector. Although several techniques are capable of revealing the slip plane and Burgers vector for a system, interpretation of three-dimensional etch pit shape (Amelinckx, 1956; Halfpenny et al., 1984), anisotropic indentation hardness (Gallagher et al., 1992), or post-growth motion of dislocations as observed by X-ray topography (Halfpenny et al., 1986) have not 
enabled the definitive identification of the slip systems for RDX. A brief review of the development of our current understanding of dislocation slip systems in RDX is given in the remainder of this section.

Surface indentation followed by etching, where material is preferentially dissolved in the vicinity of the sites where dislocations meet the surface, has been the most common technique for characterizing plastic deformation in molecular crystals. Elban and Armstrong (1982) and Elban et al. (1984, 1989) indented the $\{210\},\{120\}$, and (010) surfaces and observed etch pit alignments that they attributed to glide of dislocations with Burgers vector [100] on $\{021\}$ and (001). Halfpenny et al. (1984) indented the (210), (111), and (001) surfaces and observed well-defined rows of etch pits attributed to the glide of dislocations with Burgers vector [001] gliding on (010). Slip on $\{011\}$ and/or $\{021\}$ by dislocations with Burgers vector [100] was also inferred, but overlapping etch pits prevented an unambiguous determination of which slip plane is dominant.

Subsequent work from the same group (Gallagher et al., 1992) using Knoop indentation on the (210) surface found that the $\{021\}[100]$ slip system best accounts for the measured anisotropic hardness; however, Elban et al. (1989) previously noted the ambiguity of analyzing such hardness tests for RDX because of anisotropic cracking and the possibility of cross-slip. Ramos et al. (2009) performed nano-indentation on (210), (021), and (001) surfaces and used atomic force microscopy to analyze deformation around indentation impressions rather than etch pit analyses. This work identified not only homogeneous nucleation of dislocations under the indenter, but also evidence supporting several additional slip systems. The slip traces imaged using atomic force microscopy were consistent with the $\{011\}[100]$ and (010)[100] systems. Hence, six slip systems had been identified by mechanical measurements (i.e., indentation), five of which involve dislocations with Burgers vector [100]. These six slip systems have been used in previous crystal plasticity simulations of the indentation experiments on RDX by Clayton and Becker (2012). A smaller subset of three slip systems (i.e. $\{021\}[100]$ and (010)[001]) formed the basis of the plasticity model developed for RDX by (De et al., 2014) with application to plate impact scenarios.

The six slip systems identified from indentation tests provide an incomplete description of the deformation mechanisms of RDX. This is best illustrated by the observation of a well defined elastic-plastic response from plate impacts on (100) RDX surfaces by Hooks et al. $(2006,2011)$ even though the resolved shear stress on the six known slip systems for this orientation is zero. Evidently, additional slip systems are required to account for plastic deformation during impacts on (100) planes. Furthermore, calculations of impacts on (210) conducted as part of this work, indicate that the previously recognized six slip systems are insufficient to fully account for the corresponding relaxation observed in the wake of the elastic precursor.

The published indentation and nano-indentation experiments have studied only a small set of surface planes (those facets exhibited by solution-grown crystals) and other slip systems may be activated by indents on other crystallographic planes. Furthermore, it is possible that indentation may lead to cracking before the activation of certain slip systems, especially in pristine samples with low densities of dislocations and sources. High-rate plate impact experiments generate large shear stresses and can suppress fracture by inertial confinement. Consequently, some slip systems that might be inactive during quasi-static indentation experiments can be activated under high rate loading encountered during plate impact experiments.

X-ray topography has been widely used to determine the Burgers vectors of dislocations in pristine molecular crystals. Whereas indentation experiments inferred only dislocations with [100] and [001] Burgers vectors, the X-ray topography studies by McDermott and Phakey (1971) and Halfpenny et al. (1986) additionally observed dislocations in as-grown RDX crystals with Burgers vectors $\langle 110\rangle,\langle 011\rangle$, and [010]. All of the dislocations, except the single dislocation with Burgers vector $\langle 011\rangle$, displayed evidence of post-growth motion, indicating that they are glissile. We have added dislocations with Burgers vectors $\langle 110\rangle,\langle 011\rangle$, and [010] observed by X-ray topography to the set of dislocations inferred from indentation experiments to extend the set of slip systems employed within our crystal plasticity model.

$\mathrm{X}$-ray topography does not directly provide information on the slip planes of the dislocations. Therefore, we propose slip planes for the dislocations seen in X-ray topography based mainly on geometric arguments and the observation that dislocations tend to glide on low index planes. Hence, we assume that dislocations with Burgers vector $\mathbf{b}=\langle 110\rangle$ glide on $\{1 \overline{1} 0\}$ and $(001), \mathbf{b}=\langle 011\rangle$ glide on $\{01 \overline{1}\}$, and $\mathbf{b}=[010]$ glides on (001). The latter system is consistent with the observation of partial dislocation loops with Burgers vector parallel to [010] gliding on (001) in large-scale molecular dynamics simulations of impacts on the (111) and 
Table 1: Description of slip systems used in crystal plasticity model for $\alpha$-phase RDX.

\begin{tabular}{|c|c|c|c|}
\hline $\begin{array}{c}\text { Slip } \\
\text { System } \\
\end{array}$ & $\begin{array}{r}\text { Crystal. } \\
\text { Plane } \\
\end{array}$ & $\begin{array}{r}\text { Slip } \\
\text { Direction } \\
\end{array}$ & $\begin{array}{r}\text { Supporting } \\
\text { References }\end{array}$ \\
\hline 1 & (021) & [100] & $1,2,5-8$ \\
\hline 2 & $(0 \overline{2} 1)$ & {$[100]$} & $1,2,5-8$ \\
\hline 3 & (011) & [100] & $1,3,7,8$ \\
\hline 4 & $(0 \overline{1} 1)$ & [100] & $1,3,7,8$ \\
\hline 5 & (010) & [100] & $1,3,5,7,8$ \\
\hline 6 & $(010)$ & [001] & $1,3,6-8$ \\
\hline 7 & (001) & [010] & 4 \\
\hline 8 & (001) & [110] & 4 \\
\hline 9 & (001) & {$[1 \overline{1} 0]$} & 4 \\
\hline 10 & (110) & {$[1 \overline{1} 0]$} & 4 \\
\hline 11 & $(1 \overline{1} 0)$ & [110] & 4 \\
\hline 12 & (011) & {$[01 \overline{1}]$} & 4 \\
\hline 13 & $(01 \overline{1})$ & [011] & 4 \\
\hline
\end{tabular}

${ }^{1}$ McDermott and Phakey (1971)

${ }^{2}$ Elban and Armstrong (1982); Elban et al. (1984)

${ }^{3}$ Halfpenny et al. (1984)

${ }^{4}$ Halfpenny et al. (1986)

${ }^{5}$ Elban et al. (1989)

${ }^{6}$ Gallagher et al. (1992)

${ }^{7}$ Ramos et al. (2009)

${ }^{8}$ Clayton and Becker (2012)

(021) planes (Cawkwell et al., 2010; Ramos et al., 2010).

The entire set of slip system geometry employed in our model is listed in Table 1. Slip system identification numbers are provided for the discussion of simulation results in Section 4. The slip system plane is described by the corresponding normal vector in reciprocal lattice coordinates and the slip directions are specified with respect to lattice coordinates. Coefficients of the corresponding vectors in a Cartesian crystallographic basis are obtained using the unit cell parameters measured under ambient conditions as reported by Choi and Prince (1972), i.e. $a=13.182 \AA, b=11.574 \AA, c=10.709 \AA$.

\section{Simulations of plate impact experiments}

\subsection{Summary of previous experiments}

Several series of plate impact experiments have been conducted on single crystal RDX specimens using a single-stage light gas gun at Los Alamos National Laboratory (Hooks et al., 2006, 2011; Cawkwell et al., 2010; Ramos et al., 2010, 2014a, 2016). The typical flyer plate impact experimental configuration is depicted in Figure 1a. The flyer plate strikes the anvil imparting a compressive wave into the anvil. The impact wave continues and eventually reaches the crystal-to-window interface, whose velocity time history is measured using velocimetry. The single crystal RDX gas gun shots used different materials, as denoted in Table 2, for anvil and window depending upon the desired peak shock pressure. In some shots, an outer epoxy potting was used (as indicated in Fig. 1a), while in several shots it was omitted; all the experiments were designed with sufficiently large crystals that release waves from free surfaces do not affect the measured interface velocity during the reported time intervals. Three representative velocity time histories measured at the RDX crystal-to-window interface are plotted in Figure 1b. For these particular shots, a two-wave structure associated with the arrival of an elastic precursor wave followed by a slight relaxation during plastic 


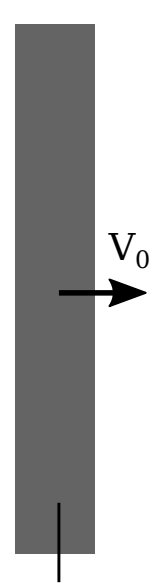

flyer plate

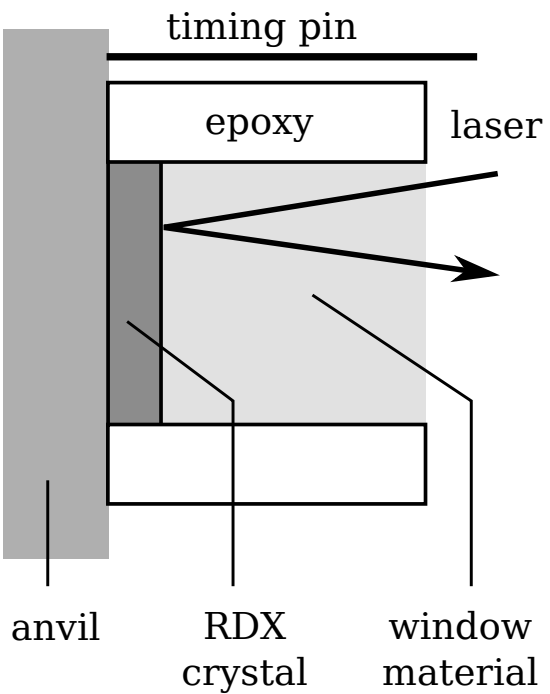

(a)

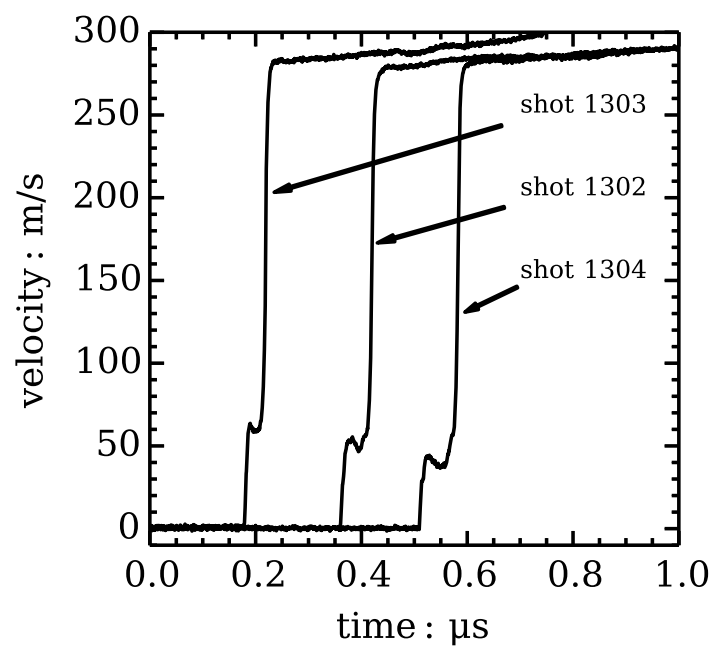

(b)

Figure 1: Illustration of plate impact experiment and diagnostics. At left (a) diagram of specimen configuration used within gas gun experiments and simulations discussed in this work. On right (b) are representative velocity time histories measured at the crystal-to-window interface using VISAR (Hooks et al., 2006, 2011).

deformation and subsequent arrival of the shock wave is evident in the velocity time history. The Hugoniot elastic limit (HEL) is related to the maximum particle velocity associated with the elastic precursor (i.e. the first wave). Table 2 summarizes 29 single crystal RDX plate impact experiments conducted at LANL. These shots collectively span peak shock pressures ranging approximately 1.1-3.0 GPa, thicknesses from under $1 \mathrm{~mm}$ to over $2 \mathrm{~mm}$, and impacts on (100), (111), (210), and (021) crystallographic planes.

RDX can undergo solid-solid phase transformations or melt at sufficiently high pressure or temperature. Dreger and Gupta (2010) determined the location of the triple point between $\alpha, \gamma$, and $\epsilon$ polymorphs of RDX at a pressure of $3.7 \mathrm{GPa}$ and a temperature of $466 \mathrm{~K}$ using Raman spectroscopy and optical imaging. At ambient pressures, the melting temperature of RDX is approximately $477 \mathrm{~K}$ (Gibbs, 1980). At temperatures and pressures below the $\alpha-\gamma-\epsilon$ triple point, RDX is stable in its $\alpha$ polymorph. There is no indication of a phase change in the velocimetry data for experiments investigated here. Moreover, our model predicts temperatures behind the shock wave for the lower (1.1-1.3 GPa) and higher (2.7-3.1 GPa) pressure impacts as 330-335 K and 370-375 K, respectively. Thus, for the experiments considered here, the material appears to remain within the solid $\alpha$ phase.

\subsection{Numerical implementation of constitutive model}

All simulations discussed here were conducted in the commercial finite element software Abaqus/Explicit and the constitutive theory has been implemented in a user subroutine (VUMAT) developed for that purpose. The implementation of the model consists of a hyperelastic stress update consistent with Eqs. $(8,15,16)$, Using subscripts $n$ and $n+1$ to indicate the value of a quantity at the end of the previous and current time increment, respectively, the time step size for a full time step is $\Delta t=t_{n+1}-t_{n}$. To limit the error accumulated in an update across time steps with a large change in deformation, a sub-incrementation scheme is used over a potentially smaller time step $\Delta t^{\star}=t_{k+1}-t_{k}$ where $t_{k+1} \leq t_{n+1}$ and $t_{k} \geq t_{n}$. A constant velocity gradient across a time step is assumed such that $\mathbf{L}=\ln \left[\mathbf{F}_{n+1} \mathbf{F}_{n}^{-1}\right] / \Delta t$. The deformation gradient at any time within the full time step is computed using the exponential map $\left.\mathbf{F}\right|_{t=t_{i}}=\mathbf{F}_{i}=\exp \left[\mathbf{L}\left(t_{i}-t_{n}\right)\right] \mathbf{F}_{n}$ in order to compute $\mathbf{F}_{k}, \mathbf{F}_{k+1 / 2}, \mathbf{F}_{k+1}$.

We define the $m^{t h}$ estimate of the plastic velocity gradient used within a Runge-Kutta scheme as

$$
\widetilde{\mathbf{L}}_{\mathrm{p}, m}=\sum_{\alpha} \dot{\gamma}_{m}^{\alpha} \mathbf{s}_{\mathrm{o}}^{\alpha} \otimes \mathbf{n}_{\mathrm{o}}^{\alpha}
$$


Table 2: Summary of experimental gas gun shots used for comparison of velocimetry with simulation results in this work.

\begin{tabular}{|c|c|c|c|c|c|c|c|c|}
\hline & & target & & peak axic & stress & & & \\
\hline shot ID & orientation & $\begin{array}{r}\text { thickness } \\
\mathrm{mm} \\
\end{array}$ & $\begin{array}{r}\text { velocity } \\
\mathrm{m} / \mathrm{s}\end{array}$ & $\begin{array}{c}\text { *experiment } \\
\text { GPa }\end{array}$ & $\begin{array}{c}{ }^{* *} \text { model } \\
\text { GPa }\end{array}$ & anvil & window & ref \\
\hline 1303 & (210) & 0.818 & 638.0 & 3.0 & 2.9 & kel-F 81 & $\mathrm{LiF}$ & 1 \\
\hline 1302 & (210) & 1.483 & 630.0 & 2.9 & 2.8 & kel-F 81 & $\mathrm{LiF}$ & 1 \\
\hline 1304 & (210) & 2.083 & 632.0 & 3.0 & 2.8 & kel-F 81 & $\mathrm{LiF}$ & 1 \\
\hline 1309 & (111) & 0.902 & 638.0 & 2.8 & 2.9 & kel-F 81 & $\mathrm{LiF}$ & 1,2 \\
\hline 1310 & (111) & 1.450 & 639.0 & 2.9 & 2.9 & kel-F 81 & $\mathrm{LiF}$ & 1,2 \\
\hline 1311 & (111) & 2.019 & 633.0 & 2.9 & 2.8 & kel-F 81 & $\mathrm{LiF}$ & 1,2 \\
\hline 1308 & (100) & 0.904 & 631.0 & 2.9 & 2.8 & kel-F 81 & $\mathrm{LiF}$ & 1 \\
\hline 1305 & (100) & 1.321 & 639.0 & 3.1 & 2.9 & kel-F 81 & $\mathrm{LiF}$ & 1 \\
\hline 1306 & (100) & 2.098 & 633.0 & 2.9 & 2.8 & kel-F 81 & $\mathrm{LiF}$ & 1 \\
\hline 1312 & (100) & 2.789 & 636.0 & 3.0 & 2.9 & kel-F 81 & $\mathrm{LiF}$ & 1 \\
\hline 1419 & (021) & 0.945 & 635.0 & 2.7 & 2.8 & kel-F 81 & PMMA & 3 \\
\hline 1420 & (021) & 1.938 & 637.0 & 2.7 & 2.8 & kel-F 81 & PMMA & 3 \\
\hline 1355 & (210) & 1.002 & 297.7 & 1.4 & 1.3 & Quartz & PMMA & 4 \\
\hline 1385 & (210) & 1.483 & 301.0 & 1.3 & 1.3 & Quartz & PMMA & 4 \\
\hline 1354 & (210) & 1.507 & 291.8 & 1.4 & 1.3 & Quartz & PMMA & 4 \\
\hline 1374 & (210) & 1.950 & 301.9 & 1.3 & 1.3 & Quartz & PMMA & 4 \\
\hline 1353 & (210) & 2.035 & 293.3 & 1.4 & 1.3 & Quartz & PMMA & 4 \\
\hline 1356 & (111) & 1.015 & - & 1.3 & 1.2 & Quartz & PMMA & 3 \\
\hline 1358 & (111) & 1.547 & 295.6 & 1.2 & 1.2 & Quartz & PMMA & 5 \\
\hline 1384 & (111) & 1.586 & 296.2 & 1.3 & 1.3 & Quartz & PMMA & 5 \\
\hline 1357 & (111) & 2.149 & 290.8 & 1.1 & 1.2 & Quartz & PMMA & 3 \\
\hline 1322 & (100) & 0.869 & 292.0 & 1.3 & 1.2 & Quartz & PMMA & 5 \\
\hline 1337 & (100) & 0.924 & 297.0 & 1.5 & 1.3 & Quartz & PMMA & 5 \\
\hline 1338 & (100) & 1.370 & 294.0 & 1.3 & 1.3 & Quartz & PMMA & 5 \\
\hline 1323 & (100) & 1.455 & 295.5 & 1.3 & 1.3 & Quartz & PMMA & 5 \\
\hline 1324 & (100) & 1.915 & 291.4 & 1.2 & 1.2 & Quartz & PMMA & 5 \\
\hline 1339 & (100) & 1.944 & 297.6 & 1.2 & 1.3 & Quartz & PMMA & 5 \\
\hline 1417 & (021) & 0.995 & 294.5 & 1.3 & 1.2 & Quartz & PMMA & 3 \\
\hline 1418 & (021) & 1.930 & 296.3 & 1.2 & 1.2 & Quartz & PMMA & 3 \\
\hline
\end{tabular}

*Approximate magnitude of peak axial compressive stress, $\left|\sigma_{11}\right|$, estimated from experimental velocimetry using impedance matching as reported in literature.

${ }^{* *}$ Magnitude of peak axial compressive stress, $\left|\sigma_{11}\right|$, obtained from simulation results using the model developed in this work. References: ${ }^{1}$ Hooks et al. (2006, 2011), ${ }^{2}$ Cawkwell et al. (2010), ${ }^{3}$ Ramos et al. (2010), ${ }^{4}$ Ramos et al. (2014a), ${ }^{5}$ Ramos et al. (2016) 
Using this expression, we compute the $m^{t h}$ estimate of the elastic deformation at time $t_{i}$

$$
\mathbf{F}_{\mathrm{e}, m}\left(t=t_{i}\right)=\mathbf{F}_{i} \mathbf{F}_{\mathrm{p}, k}^{-1} \exp \left[-\widetilde{\mathbf{L}}_{\mathrm{p}, m}\left(t_{i}-t_{k}\right)\right]
$$

Corresponding to $\mathbf{F}_{\mathrm{e}, m}^{i}$ is a hyperelastic estimate of stress $\boldsymbol{\sigma}_{m}^{i}$ consistent with the nonlinear thermoelastic description of the material. A fourth-order Runge-Kutta scheme is used to evaluate the slip and dislocation evolution rates at the mid-point of the subincrement, i.e.,

$$
\dot{\gamma}_{k+\frac{1}{2}}=\frac{1}{6}\left(\dot{\gamma}_{1}+2\left(\dot{\gamma}_{2}+\dot{\gamma}_{3}\right)+\dot{\gamma}_{4}\right) \quad \text { and } \quad \dot{\varrho}_{k+\frac{1}{2}}=\frac{1}{6}\left(\dot{\varrho}_{1}+2\left(\dot{\varrho}_{2}+\dot{\varrho}_{3}\right)+\dot{\varrho}_{4}\right)
$$

where the intermediate rates are evaluated according to a standard $4^{\text {th }}$ order Runge-Kutta scheme. The error in slip rates associated with the integration over a subincrement is estimated as the difference between the fourth-order Runge-Kutta estimate and a third-order estimate of the slip rates,

$$
\text { Error } \approx\left|\dot{\gamma}_{k+\frac{1}{2}}-\dot{\gamma}_{k+\frac{1}{2}}^{R K 3}\right| /\left|\dot{\gamma}_{k+\frac{1}{2}}+\epsilon\right|
$$

where $\epsilon$ is a small number and the third-order estimate of slip rate is evaluated according to $\dot{\gamma}^{R K 3}$ is obtained from a standard $3^{\text {rd }}$ order Runge-Kutta scheme. If the estimated error associated with the subincrement is less than $10^{-3}$, then the update over the subincrement is computed according to

$$
\mathbf{F}_{\mathrm{p}, k+1}=\exp \left[\Delta t^{\star} \widetilde{\mathbf{L}}_{\mathrm{p}, k+\frac{1}{2}}\right] \mathbf{F}_{\mathrm{p}, k} \quad \text { and } \quad \mathbf{F}_{\mathrm{e}, k+1}=\mathbf{F}_{k+1} \mathbf{F}_{\mathrm{p}, k+1}^{-1}
$$

where $\widetilde{\mathbf{L}}_{\mathrm{p}, k+\frac{1}{2}}$ is evaluated by substituting $\dot{\gamma}_{m}^{\alpha}=\dot{\gamma}_{k+1 / 2}^{\alpha}$ into Eq. 38. If the error is larger than the specified tolerance, the subincremental time step $\Delta t^{\star}$ is reduced and the subincrement is attempted again. The sub-incrementation scheme is repeated until an update across the entire time step $\Delta t$ is complete.

The temperature is held fixed at $T=T_{n}$ for the sub-incremental update of crystal plasticity and then explicitly integrated over a full time step using according to $T_{n+1}=T_{n}+\dot{T} \Delta t$ where $\dot{T}$ is evaluated according to Eq. 9 assuming adiabatic conditions, i.e., zero heat flux. Note that the energy storage due to evolving dislocation fields within Eq. 10 is approximated as $\sum_{\alpha} f^{\alpha} \dot{\varrho}^{\alpha}=\beta \mathcal{P}_{p}$ using a constant energy storage coefficient, $\beta$. The fraction of plastic work converted to heat during deformation of RDX has not been previously investigated; in the absence of detailed investigation, we assign the value $\beta=0.05$ in these calculations. The specific heat at constant volume is evaluated from the free energy at the current state, i.e., $C_{V}=T \frac{\partial s}{\partial T}$.

\subsection{Finite element model of one-dimensional plate impact problem}

Simulations of one-dimensional (1D) plate impact were conducted by using a 1D array of three-dimensional hexahedral elements (employing linear interpolation of displacement field and reduced integration) along the shock direction. In "full" geometry calculations, the flyer plate is directly included and is discretized into 1,000 elements along its thickness. Only $4 \mathrm{~mm}$ of the entire $12 \mathrm{~mm}$ thick window is included in these calculations, since that travel distance provides sufficient time for a shock wave to transit from the RDX-window interface to a free surface (and return as rarefaction wave) without affecting the interface velocity. The anvil and window are resolved using 1,000 and 800 elements, respectively, while the number of elements throughout the RDX specimen was varied in order to attain an element size of approximately $5 \mu \mathrm{m}$. The constitutive behavior of the flyer, anvil, and window materials was specified using Mie-Grüneisen equations of state and perfect plasticity, where applicable, in accordance with parameters tabulated by Steinberg et al. (1996). A Mie-Grüneisen equation of state was also used for the polymethyl methacrylate (PMMA) windows, along with a tabulation of a cubic fit to shock velocity, $U_{s}$, versus particle velocity, $U_{p}$, data over the range $0<U_{p}<540 \mathrm{~m} / \mathrm{s}$ previously reported by Schuler (1970) and Schuler and Nunziato (1974).

Contact was modeled between the flyer plate and anvil impact interface, although separation of the flyer from anvil occurs sufficiently late in the experiment that it does not affect the window interface velocity during the recorded interval. Relative transverse slip is allowed at both the anvil-to-specimen and specimento-window interfaces in order to enable transverse components of waves in the RDX without accounting 
for friction nor more general constitutive behavior to capture shearing within the anvil or window materials. Periodic displacement boundary conditions are enforced along the length of the model to enable both longitudinal and transverse components of deformation associated with generalized 1D plane waves. Initial conditions consist of the anvil/target/window at rest while nodes of the flyer plate are assigned an initial velocity based on the experimentally measured flyer velocity for each shot. All simulations use a uniform initial temperature of $T=300 \mathrm{~K}$.

We note here several important aspects of these calculations that are necessary to correctly compute RDX crystal-to-window interface velocity consistent with the measurements in contrast with a previous approach by De et al. (2014). Firstly, accounting for the anvil material is important to translate the measured flyer plate impact velocity into the appropriate particle velocity time history at the anvil-to-RDX interface. Instead of directly modeling the propagation of the compressive wave throughout the experimental configuration, De et al. (2014) directly applied a pressure to the surface of the RDX specimen. However, pressure was not directly measured in any of the experiments, thus De et al. (2014) specified a ramp from zero to the estimated peak shock pressures as reported by Hooks et al. (2006) over a time interval of $1 \mu \mathrm{s}$. Note, the characteristic time associated with the actual interface pressure between anvil and RDX is much lower, e.g. O(5-50 ns). Presumably, this slow ramp in pressure could be a contributor to the diffuse nature of the elastic precursor observed in their previous simulations (cf. De et al., 2014, Figs. 3-5) when compared with experiment.

Secondly, we note the importance of including the window in these calculations in order to correctly compute RDX crystal-to-window interface velocity consistent with the measurements. Previous work by De et al. (2014) omitted the window, instead modeling a $3 \mathrm{~mm}$ thickness of RDX specimen (i.e. larger than that for any of the actual experiments). Rather than computing RDX-to-window interface velocity results by modeling each shot separately, they apparently conducted a single simulation and extracted particle velocity time histories at several locations corresponding to the measured specimen thicknesses. This approach effectively treats the window as though comprised of RDX rather than the representing the differing shock impedance of the actual window material.

Details of the nodal boundary conditions employed in De et al. (2014) along the faces orthogonal to the direction of loading are unclear from that manuscript. We note that in our simulation results suppressing transverse displacements affects the precursor magnitude for lower-pressure $(\approx 1.25 \mathrm{GPa})$ impact on $(111)$.

Finally, the previous work by De et al. (2014) produces simulation results that seem to be in reasonable agreement with velocimetry obtained at a crystal-to-window interface from the data published by Hooks et al. (2006). However, Hooks et al. (2011) published an erratum to correct the velocity time histories previously published by Hooks et al. (2006); the latter source should not be used for quantitative comparisons since the window correction factor was inadvertently applied twice during analysis of the raw data. The simulation results of De et al. (2014) do not match the corrected velocimetry results appearing in Hooks et al. (2011).

\subsection{Calibration of model parameters}

Parameter values used within our single crystal model for RDX are listed in Tables 3-5. Many thermoelastic properties of explosive molecular crystals have been measured and calculated using a variety of techniques near room temperature conditions (cf. Hooks et al., 2015; Bolme and Ramos, 2014; Taylor, 2014). We use parameters for the free energy-based EOS for $\alpha$-RDX from Cawkwell et al. (2016), values for the elastic constants and their temperature derivatives reported by Haussühl (2001) and the corresponding pressure derivatives calculated by Sewell and Bennett (2000) for $\alpha$-RDX, as included in Table 3. Table 4 lists coefficients for a quadratic variation of stress-free thermal strain obtained by fits to temperature dependent coefficients of thermal expansion presented by Cady (1972). Note, the elastic moduli reported here and used in this work are obtained from measurements and simulations that are more nearly isentropic rather than isothermal. The difference between isothermal and isentropic moduli for RDX are on the order of $500 \mathrm{MPa}$ at room temperature (cf. Clayton and Becker, 2012), which is smaller than the discrepancy in the reported parameters from several sources as discussed by Bolme and Ramos (2014). For this reason, we do not distinguish between isentropic and isothermal elastic constants in this work.

Values for parameters in the plasticity model, listed in Table 5, are motivated by physical arguments and/or simplifying approximations in most cases. For example, rather than computing the shear modulus for 
each slip system, we assign an effective value that coarsely applies to all slip systems. While the magnitude of the Burgers vector is different across families of slip systems, we employ a single order-of-magnitude approximation for Burgers vector magnitude pertaining to all slip systems as $10 \AA$. The attempt frequency for thermally-activated fluctuations past obstacles to dislocation motion is related to the frequency of the lattice phonons. We approximate this attempt frequency using the Debye temperature of the lattice phonons consistent with the equation of state, i.e. $f_{D}=k_{\mathrm{B}} T_{\mathrm{D}, 1} / h$, where $h$ is the Planck constant and $T_{\mathrm{D}, 1}=188.8 K$ is the Debye temperature corresponding to lattice phonons (Cawkwell et al., 2016), which gives approximately $f_{D}=4 \mathrm{THz}$.

The seven plasticity parameters appearing below the horizontal line in Table 5 were calibrated to bring simulated velocity results into agreement with velocimetry data measured in a plate impact experiment. Results from a single experimental shot (i.e. 1302) producing an approximately 2.9 GPa shock pressure with impact on the (210) crystallographic plane on an approximately $1.5 \mathrm{~mm}$ thick target specimen were used for the calibration. Parameter sensitivities were investigated in order to develop an understanding of the effects of the various plasticity parameters in producing changes in various features of the velocimetry response. An initial simulation of shot 1302 included the 'full' problem geometry described previously in Section 4.3. Velocity time history results at the anvil-to-crystal interface were computed and stored for a nominal selection of parameters with trial values for the undetermined plasticity parameters. Subsequent calibration simulations of shot 1302 were conducted using only 'partial' geometry by omitting the flyer plate and anvil mesh and instead specifying the previously simulated anvil-target interface velocity as a boundary condition at that location. While the effective impedance between anvil and target depends on the details of the plasticity model, parameter variations were not observed to significantly modify the simulated particle velocity time history at this interface. Note, the window was retained in these calculations to ensure their effect on interface velocity was included for comparison with the experimental observation. Since we parameterize the plasticity model to one plate impact experiment, we are truly able to assess its ability to predict the response of crystals of different thicknesses and orientations under different impact conditions. If we had used all available data sets to parameterize the model and included more detailed descriptions, for example of system specific slip resistances, it would be more difficult to assess the predictive capabilities of the model.

Parameter values for initial athermal slip resistance and initial density of mobile dislocations identified through our calibration are consistent with experimental measurements. Ramos et al. $(2009,2011)$ measured deformation consistent with dislocation source activation and nucleation at maximum resolved shear stresses ranging from 400 to $900 \mathrm{MPa}$. Halfpenny et al. (1984) measured dislocation densities ranging from 10 to $10^{3} \mathrm{~cm}^{-2}$ in RDX grown from acetone, and it is reasonable to expect that the density would increase by a few orders of magnitude during the cutting and polishing of our samples that were similarly grown from acetone.

The effects of varying the initial mobile dislocation density and the dislocation multiplication coefficient on the resulting simulated RDX specimen-to-window interface are illustrated in Figure 2. Increasing the initial dislocation density decreases the height of the elastic precursor by enabling deformation to be more readily accommodated through plastic slip upon arrival of the stress wave. Likewise, increasing the dislocation multiplication rate allows dislocations to be generated more rapidly early on thus reducing the elastic precursor magnitude. On the other hand, lower values of dislocation multiplication coefficient increase the plastic rise time and the separation between elastic precursor and plastic wave. We note that, in comparison with typical ranges of $0.01<C_{\mathrm{M}}<0.1$ used for metallic single crystals (cf. Ma and Roters, 2004; Ma et al., 2006; Austin and McDowell, 2011), the calibrated value of $C_{\mathrm{M}}=4$ is quite large. As discussed in Section 3 , we propose that the multitude of available slip planes for dislocations of Burgers vector [100] in $\alpha$-RDX facilitates easy cross slip, and double cross slip in particular, thus leading to the rapid generation of prolific Frank-Read sources accounting for our relatively large coefficient.

An approximate saturation value of dislocation density is related to the multiplication and annihilation coefficients via Equation 32. Figure 3 illustrates the effects of varying this approximate saturation value for mobile dislocation density as well as the ratio of the trapping coefficient to multiplication coefficient. The shot at higher pressure (i.e. 1302) shows less sensitivity to variations in these parameters than does the shot 
Table 3: Elastic coefficients and their temperature and pressure derivatives used in simulations. Note, the values reported by (Haussühl, 2001) and (Sewell and Bennett, 2000), measured under conditions more nearly isentropic than isothermal, are used directly in this work.

\begin{tabular}{rrrr}
\hline component & $C_{i j}^{\text {ref }}$ & $\frac{\partial C_{i j}}{\partial T}$ & $\frac{\partial C_{i j}}{\partial P}$ \\
$(i, j)$ & $\mathrm{GPa}$ & $\mathrm{MPa}-K^{-1}$ & 15.74 \\
\hline$(1,1)$ & 24.98 & -37.22 & 17.87 \\
$(2,2)$ & 19.58 & -30.35 & 13.17 \\
$(3,3)$ & 17.89 & -33.63 & 4.09 \\
$(4,4)$ & 5.15 & -9.79 & 2.72 \\
$(5,5)$ & 4.06 & -5.85 & 4.23 \\
$(6,6)$ & 6.90 & -10.28 & 7.35 \\
$(2,3)$ & 5.90 & -6.49 & 6.25 \\
$(3,1)$ & 5.80 & -6.96 & 6.93 \\
$(1,2)$ & 8.19 & -2.46 & \\
\hline
\end{tabular}

Table 4: Coefficients used for quadratic expression of thermal strain, i.e. $\boldsymbol{\alpha}=\boldsymbol{\alpha}^{a}\left(T-T^{\mathrm{ref}}\right)+\boldsymbol{\alpha}^{b}\left(T-T^{\mathrm{ref}}\right)^{2}$ obtained from measurements of Cady (1972) where $T^{\mathrm{ref}}=300 \mathrm{~K}$

\begin{tabular}{rrr}
\hline component $(i, j)$ & $\alpha_{i j}^{\mathrm{a}}\left(\mathrm{K}^{-1}\right)$ & $\alpha_{i j}^{\mathrm{b}}\left(\mathrm{K}^{-2}\right)$ \\
\hline$(1,1)$ & $2.80 \times 10^{-5}$ & $4.24 \times 10^{-8}$ \\
$(2,2)$ & $8.66 \times 10^{-5}$ & $2.57 \times 10^{-8}$ \\
$(3,3)$ & $8.47 \times 10^{-5}$ & $12.78 \times 10^{-8}$ \\
\hline
\end{tabular}

at lower pressure (i.e. 1338). It is clear from the latter cases that decreasing the approximate saturation value for mobile dislocation density decreases the slope of the plastic rise. Increasing the dislocation trapping coefficient has a similar effect on the slope of the plastic rise, but also increases the magnitude of the elastic precursor height, because fewer of the initially mobile dislocations remain mobile to accommodate early plasticity.

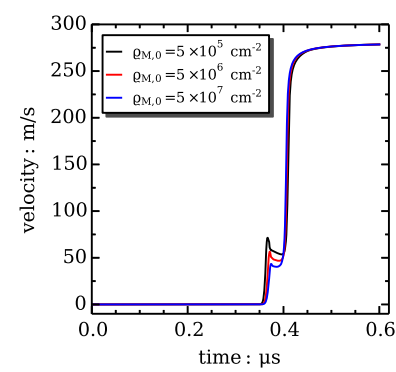

(a) Shot $1302, \varrho_{M, 0}$

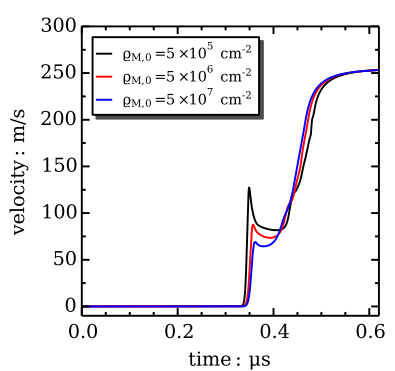

(b) Shot $1338, \varrho_{M, 0}$

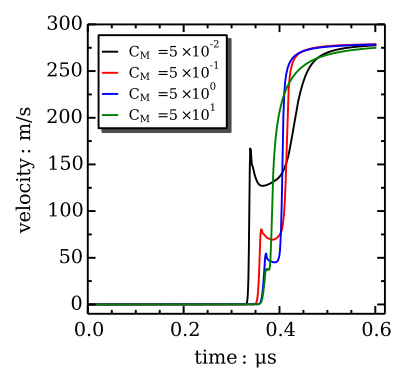

(c) Shot $1302, C_{M}$

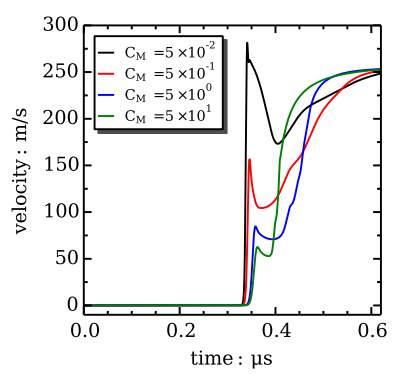

(d) Shot $1338, C_{M}$

Figure 2: Relative effects of varying (cf. a,b) initial mobile dislocation density and (cf. c,d) the dislocation multiplication rate coefficient on simulated RDX/window interface velocity. 
Table 5: Parameter values for plasticity model used in this work. The seven parameters below the horizontal line were varied in order to fit model results to experiment for shot 1302.

\begin{tabular}{llll}
\hline Parameter & Value & Unit & Description \\
\hline$\mu$ & 6.0 & GPa & effective shear modulus \\
$|\mathbf{b}|$ & 10 & $\AA$ & Burgers vector length \\
$\bar{L}$ & $10|\mathbf{b}|$ & $\AA$ & mean barrier spacing \\
$f_{D}$ & $4 \times 10^{6}$ & $\mathrm{MHz}$ & attempt frequency \\
$q_{1}$ & 0.33 & - & barrier shape parameter \\
$q_{2}$ & 1.66 & - & barrier shape parameter \\
$Y_{e}$ & $6|\mathbf{b}|$ & $\AA$ & dislocation capture distance \\
$H_{\alpha \beta}$ & 0.25 & - & dislocation-dislocation interaction matrix \\
\hline$B_{0}$ & $3 \times 10^{-4}$ & $\mathrm{MPa}-\mu \mathrm{s}$ & dislocation phonon drag viscosity \\
$C_{\mathrm{M}}$ & 4 & - & dislocation multiplication coefficient \\
$C_{\mathrm{A}}$ & 0 & - & dislocation annihilation coefficient \\
$C_{\mathrm{T}}$ & 0 & - & dislocation trapping coefficient \\
$\tau_{0}$ & 540 & $\mathrm{MPa}$ & initial athermal slip resistance (Peierls stress) \\
$\varrho_{0}$ & 0.05 & $\mu \mathrm{m}^{-2}$ & initial density of mobile dislocations \\
$g_{0}$ & 0.025 & - & dislocation energy barrier coefficient \\
\hline
\end{tabular}

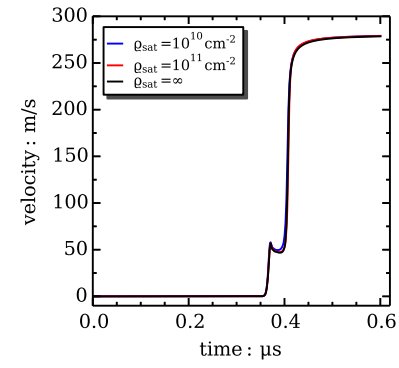

(a) Shot 1302, $\varrho_{\text {sat }}$

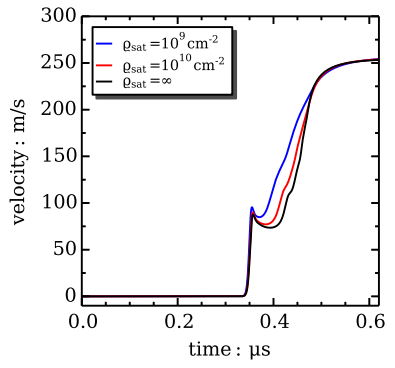

(b) Shot $1338, \varrho_{\text {sat }}$

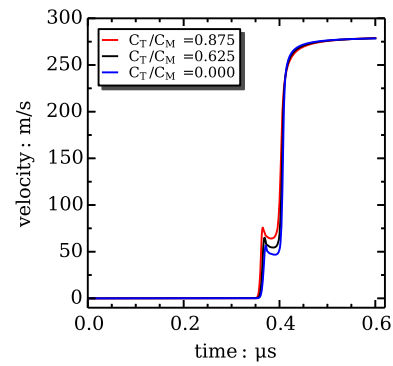

(c) Shot $1302, C_{T}$

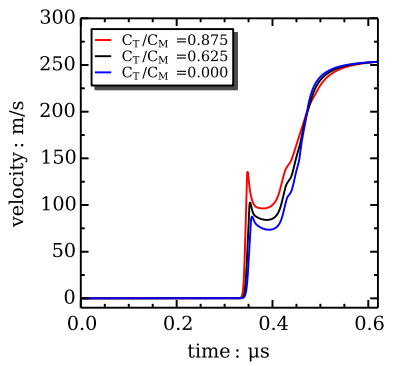

(d) Shot $1338, C_{T}$

Figure 3: Relative effects of varying (cf. a,b) the saturation value of dislocation density and (cf. c,d) the dislocation trapping coefficient on simulated RDX/window interface velocity. 


\subsection{Comparison of simulation results with plate impact experiments}

Plots comparing the simulated RDX/window interface velocity with corresponding measurements from all flyer plate impact experiments listed in Table 2 are presented in Figures 4-7. In these plots, red and black lines correspond to simulation and experimental results, respectively. These figures are arranged such that the lower shock pressure cases are included in subfigure (a) on the left and higher shock pressures in subfigure (b) on the right. The experiment and simulation results are plotted with respect to the time the compression wave reaches the anvil/RDX interface; time axes have not been shifted to achieve a better alignment or to de-clutter the figures. Arrival times from simulation are typically in agreement with experiment to within $25 \mathrm{~ns}$, but up to $50 \mathrm{~ns}$ in a few cases. This is likely an artifact of experimental uncertainties (Ramos et al., 2016). Table 6 identifies the active slip systems based on simulation results for each crystal orientation considered here.

All shots with impacts on (210) are represented in Figure 4. Considering that the parameters in the plasticity model were adjusted to calibrate simulation results with shot 1302 , i.e. the intermediate thickness, higher-pressure case with impact on (210), it is reassuring that the simulation results for all three higherpressure cases are in good agreement with the experimental data for this orientation. The model captures the elastic and plastic wave speeds as evidenced by the increase in separation of elastic and plastic waves for thicker specimens. The model also captures the magnitude of the elastic precursor wave and its decrease with increasing specimen thickness. The lower pressure shots exhibit a slower, more gradual rise associated with the plastic wave and the model captures this effect also. There are two lower-pressure shots that were conducted at the (similar) thickest target dimensions. The magnitudes of elastic precursor waves observed in simulation results of these larger specimen thicknesses are between those observed from the corresponding experimental results.

Experiments with line imaging velocimetry (Ramos et al., 2014a) recorded similar spatial variations in velocity histories for single samples in a single experiment that were attributed to spatially inhomogeneous dislocation densities. Because of the large sensitivity of the simulation results to the initial dislocation density and early dislocation generation, we speculate that experimental observations would likely exhibit some variability that may be attributed to spatial and/or specimen-to-specimen variations of initial defect densities. Based partly on this speculation, extensive efforts to improve these fits are deemed unwarranted prior to in the absence of further experimental insight.

All shots with impact on (100) are included in Figure 5. The six slip systems used in earlier studies have zero resolved shear stress for impacts on (100) and cannot account for the elastoplastic behavior seen in the experimental velocimetry results. Using the additional slip systems proposed in Section 3.1, which have non-zero resolved shear stresses for this orientation, the model represents plasticity during impact on (100) reasonably well. The elastic and plastic wave arrival times and plastic rise times observed in simulation and experimental results coincide for the lower pressure shots. The simulation results are in strong agreement with experimental results for the thinnest and intermediate thickness specimens subjected to lower shock pressures. The thickest specimens under lower shock pressure exhibit a larger magnitude of elastic precursor wave than the simulation results. Furthermore, the magnitude of the elastic precursor evident in experimental data is larger for the thickest than for any of the thinner specimens. In this case, the model is consistent with the typically observed trend of decaying magnitude of elastic precursor wave with increasing propagation distance. The larger precursor magnitude (for thickest specimens) is observed in the two shots at approximately the same specimen thickness; thus, it is plausible that this is not an anomaly. The agreement between simulation and experimental results for the higher pressure impacts normal to (100) show reasonable agreement. Note, the experimental results for shots with impact along (100) were not used for calibrating model parameters.

All shots with impact on (111) are presented in Figure 6. In this case, we see similar agreement between experiment and simulation. For the lower pressure cases, the model exhibits a faster plastic rise (i.e. a steeper slope for the secondary wave) than the experiment. Based on model parameter sensitivity studies, it is plausible that this discrepancy may correspond to saturation of the evolving dislocation density in experiment. Simulation results for the higher pressure (111) shots match experimental results exceptionally well. There is a $50 \mathrm{~ns}$ discrepancy in the arrival time of the wave for the thinnest specimen that is consistent 
with experimental uncertainty.

For both the (111) and (021) (cf. Figure 7) orientations, the distinct two-wave structure evident at lower pressures is overdriven into a single shock wave for the higher pressure shots. Our single crystal plasticity model accurately predicts both the distinct elastic and plastic waves for (100) and (210) oriented RDX single crystals and the overdriven response of the (111) and (021) oriented crystals at $3 \mathrm{GPa}$, respectively.

Previous work proposed that the overdriven responses observed for $P \approx 3$ GPa impacts on (111) and (021) arise from an orientation-dependent hardening mechanism (Cawkwell et al., 2010; Ramos et al., 2010). Largescale molecular dynamics simulations showed that partial dislocation loops with Burgers vectors parallel to [010] homogeneously nucleate and expand on (001) in the vicinity of the shock front during impacts on (111) above a threshold shock pressure. These expanding partial dislocation loops rapidly generate a high density of stacking faults that were anticipated to plastically harden the material, thus potentially explaining the previously unexpected, 'anomalous' response observed experimentally. Based on the orientation dependence of the [010](001) slip system it was predicted that impacts on (021) would give rise to the same response, i.e., a two-wave response at low pressure and an apparently overdriven response at pressures around $3 \mathrm{GPa}$ (Cawkwell et al., 2010). The predicted response was confirmed experimentally (Ramos et al., 2010). Our model does not include this hardening mechanism. Thus, the results in Figures $6 \mathrm{~b}$ and $7 \mathrm{~b}$ provide for a more satisfactory explanation of the 'anomalous' response as being, instead, a natural consequence of the elastic and plastic anisotropy of the material.

Our single crystal plasticity model shows that the [010](001) slip system is active for impacts on (111) and (021), but with perfect lattice dislocations rather than partial dislocations. We performed highly accurate density functional theory calculations to assess the stability of the stacking faults seen in the earlier largescale MD simulations. These calculations verified the determination from previous MD simulations (Cawkwell et al., 2010), namely that the stacking fault on (001) is stabilized by a change in the conformation of the molecules at the fault plane when the stress normal to (001) is sufficiently large. Hence, the stacking faults seen in Cawkwell et al. (2010) should not be dismissed as being unphysical artifacts of the Smith-Bharadwaj potential used in those calculations. We instead suggest that the stacking faults may play a role in the dissociation of [010] dislocations into partials on (001).

The slip systems included in our single crystal plasticity model evidently provide a good description of the anisotropic elastic-plastic response of RDX. We have tried to establish a minimal set of slip systems that is capable of representing the shock response of RDX by removing from the model some of the seven additional slip systems proposed here. However, in every attempt, the agreement between the predictions of the model and experiment became worse with respect to the full model with 13 slip systems. However, we have no external justification that our set of slip systems is either complete or unique since the slip planes for the dislocations identified by X-ray topography were proposed based on geometric arguments. Further experiments including indentation, X-ray topography, and in situ X-ray diffraction will be necessary to assess the validity of the set of slip systems proposed here for high strain rate deformation.

RDX is a brittle material characterized by relatively easy fracture under certain loading cases (Elban et al., 1989; Sharma et al., 2001). Nevertheless, fracture can be suppressed in shock and well-controlled indentation experiments owing to the compressive loading combined with inertial confinement. While fracture is a plausible mechanism, it is evident from our simulation results that the shock responses of RDX crystals are captured well without including fracture in the framework. We note that the velocimetry data do not exhibit large stochastic variation from the model that one might expect if the actual deformation process were dominated by fracture.

\subsection{Evolution of material state across shock wave}

Figures 8-12 depict the spatial distribution of several state and driving force variables across the shock wave. In each of these plots, the position axis is measured with respect to the wave front, i.e., negative position values indicate the distance behind the leading edge of the stress wave. In Figure 8, the accumulated slip for each active slip system is plotted for (a) shot 1357 and (b) shot 1311 with impact normal to (210) crystal planes. In the (a) lower pressure shot (i.e. 1357), the plastic deformation does not begin to accumulate substantial slip until approximately $200 \mu \mathrm{m}$ behind the wave front. Also, the timing of plastic evolution on 


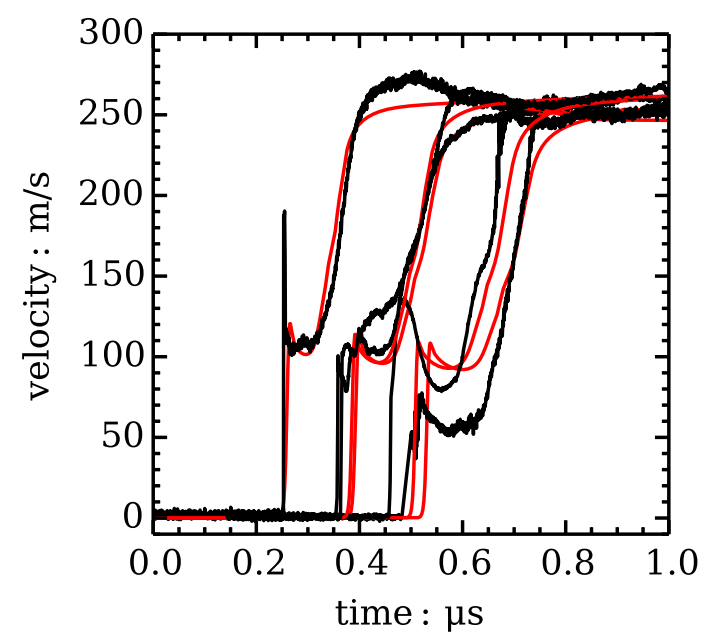

(a) shock pressure $\approx 1.3 \mathrm{GPa}$

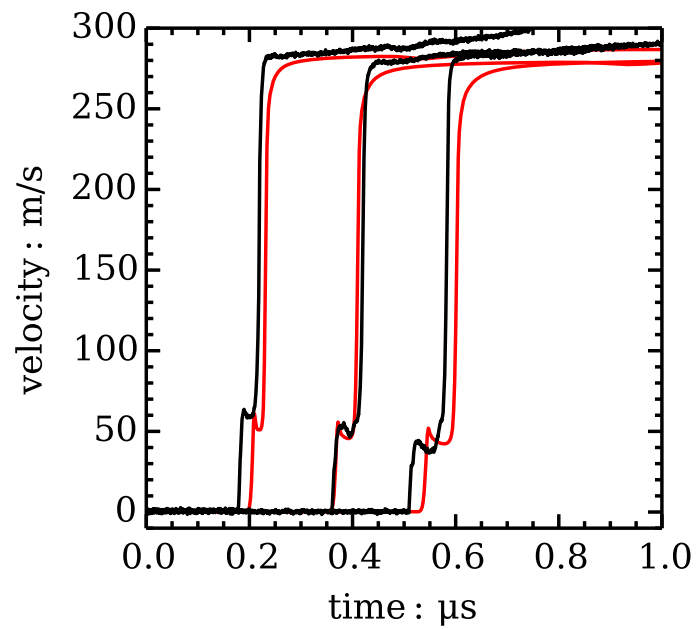

(b) shock pressure $\approx 3.0 \mathrm{GPa}$

Figure 4: Comparison of RDX crystal-window interface velocity time history results from simulation (shown in red) with those measured in corresponding experiments (shown in black) for impact normal to (210) crystallographic planes.

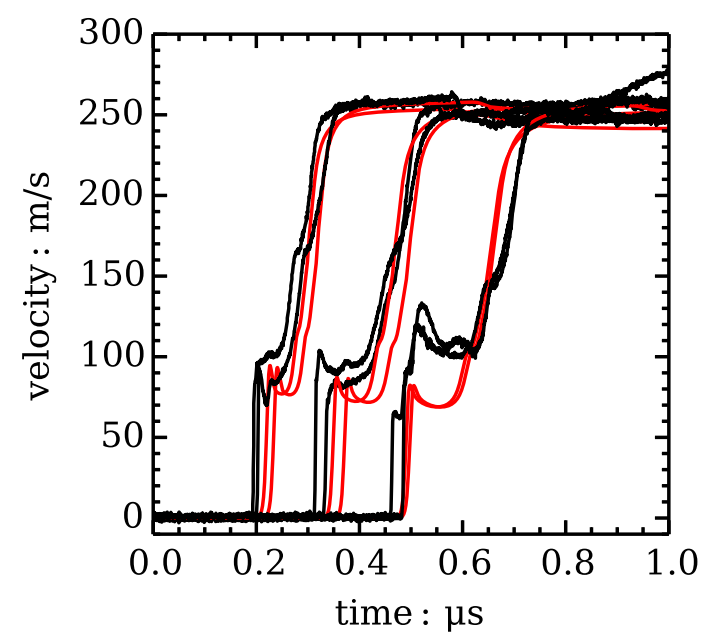

(a) shock pressure $\approx 1.3 \mathrm{GPa}$

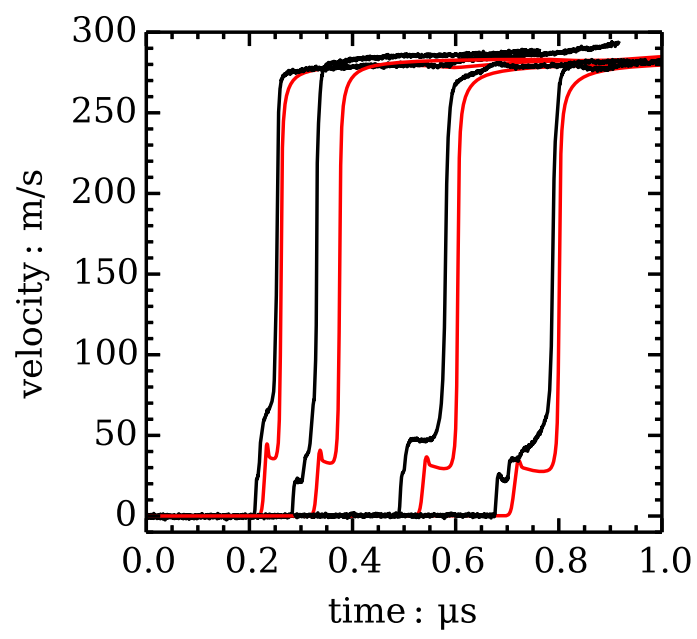

(b) shock pressure $\approx 3.0 \mathrm{GPa}$

Figure 5: Comparison of RDX crystal-window interface velocity time history results from simulation (shown in red) with those measured in corresponding experiments (shown in black) for impact normal to (100) crystallographic planes. 


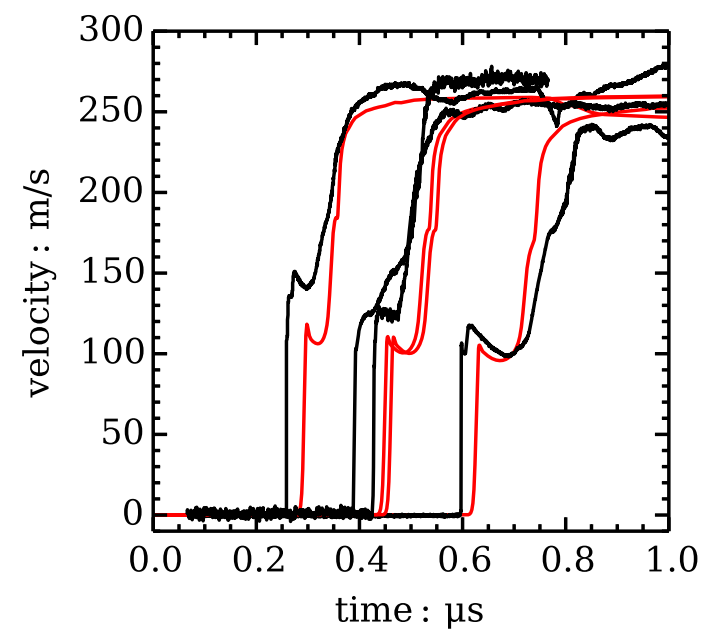

(a) shock pressure $\approx 1.3 \mathrm{GPa}$

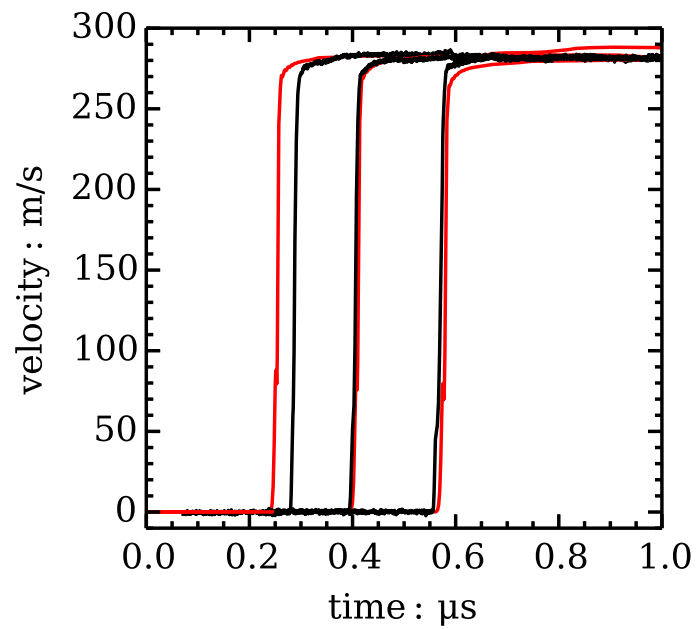

(b) shock pressure $\approx 3.0 \mathrm{GPa}$

Figure 6: Comparison of RDX crystal-window interface velocity time history results from simulation (shown in red) with those measured in corresponding experiments (shown in black) for impact normal to (111) crystallographic planes.

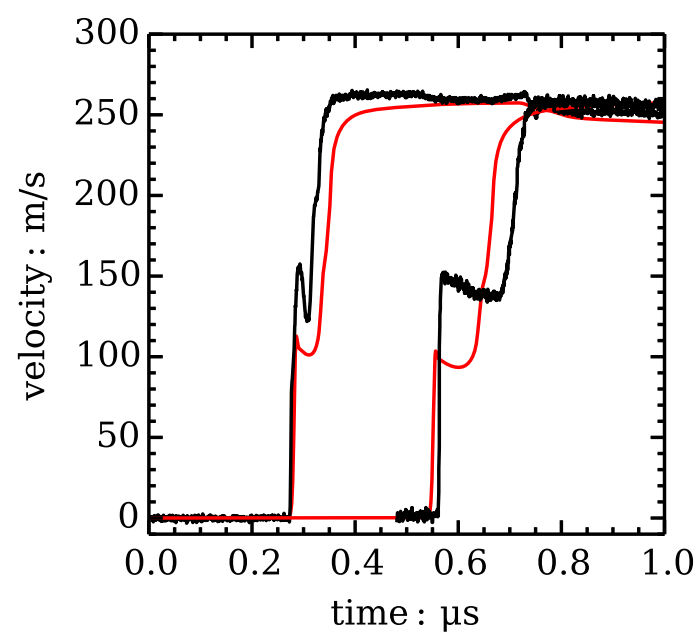

(a) shock pressure $\approx 1.3 \mathrm{GPa}$

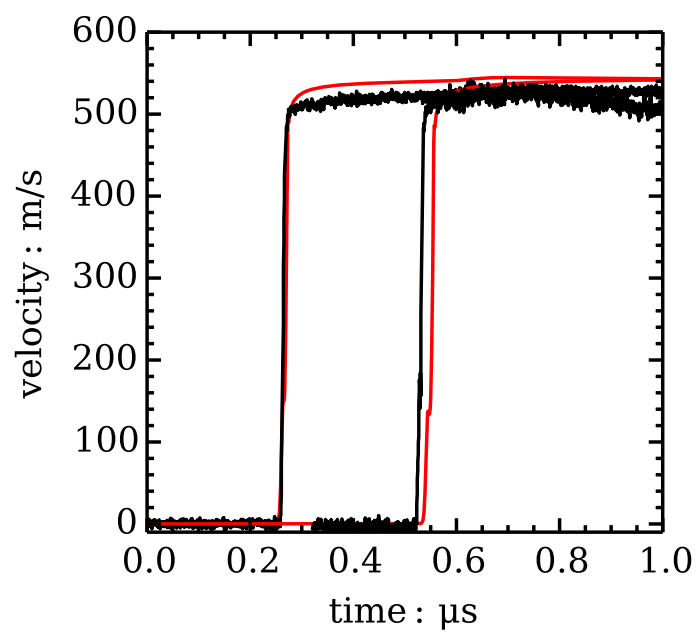

(b) shock pressure $\approx 3.0 \mathrm{GPa}$

Figure 7: Comparison of RDX crystal-window interface velocity time history results from simulation (shown in red) with those measured in corresponding experiments (shown in black) for impact normal to (021) crystallographic planes. 
Table 6: Dominant slip system activity observed in simulations for crystal orientations impacted during experiments.

\begin{tabular}{lrrrr}
\hline & \multicolumn{4}{c}{ Active Slip Systems } \\
Orientation & $(210)$ & $(100)$ & $(111)$ & $(021)$ \\
\hline & $(010)[100]$ & $(110)[1 \overline{1} 0]$ & $(021)[100]$ & ${ }^{2}(001)[010]$ \\
& $(110)[1 \overline{1} 0]$ & $(1 \overline{1} 0)[110]$ & $(001)[110]$ & $(110)[1 \overline{1} 0]$ \\
& $(011)[01 \overline{1}]$ & $(011)[01 \overline{1}]$ & $1,2(010)[001]$ & $(1 \overline{1} 0)[110]$ \\
& $(01 \overline{1})[011]$ & $(01 \overline{1})[011]$ & $1(001)[010]$ & $(011)[01 \overline{1}]$ \\
& & ${ }^{1}(011)[100]$ & \\
\hline
\end{tabular}

${ }^{1}$ Slip systems with non-negligible, but much smaller cumulative slip activity

${ }^{2}$ Slip systems associated with previous hypotheses on anomalous hardening

different slip systems is not coincident; slip on $\{011\}\langle 01 \overline{1}\rangle$ initiates at least $50 \mu \mathrm{m}$ behind slip on (010)[100] and (110)[110]. At the (b) higher shock pressure of shot 1302, substantial plastic slip accumulation occurs approximately $100 \mu \mathrm{m}$ behind the leading edge of the wave. The distance between significant slip activity on $\{011\}\langle 01 \overline{1}\rangle$ and (010)[100] plus (110)[110] is similarly compressed in comparison with the (a) lower pressure shot. The spatial profiles of the (a) projected shear stress and (b) dislocation velocity corresponding to shot 1302 at the same time as Figure 8b for the relevant slip systems are compared in Figure 9. Again, there is a delay in dislocation activity on $\{011\}\langle 01 \overline{1}\rangle$ attributed to temporary relaxation and subsequent reloading, and in correspondence with delayed accumulation of slip on that plane, evident in Fig. 8. As discussed below, the early slip activity on (010)[100] plus (110)[110] relaxes the stresses on $\{011\}\langle 01 \overline{1}\rangle$ until further deformation is not accommodated by plasticity on the former systems and stress on the later systems continues to rise. While there is significant driving stress and initially significant dislocation velocity on $\{021\}[100]$, an insufficient number of dislocations are generated to cause appreciable slip on these systems, thus the dotted lines in Figure 8 indicate negligible activity for these systems under impact normal to (210).

There are three possible causes of a shift in the dominantly active slip system like that exhibited in Fig. 9: i. Delayed arrival of stress components that have a higher projection onto $\{011\}$ slip planes due to the difference in wave mode velocities (i.e. longitudinal, transverse, or quasi modes depending upon orientation), ii. reorientation of slip systems or iii. evolution of stress due to slip activity on primary systems.

Assuming small deformation and linear elastic behavior facilitates an approximate analysis of the characteristics of propagating elastic waves. Eigenvalue solutions of the Christoffel equation, $\left(\mathbf{I} \rho c^{2}-\mathbf{n} \cdot \mathbb{C} \cdot \mathbf{n}\right) \mathbf{u}=$ $\mathbf{0}$, for impacts on RDX are listed in Table 7 . The direction of wave propagation, $\mathbf{n}$, is along the specimen $X$-axis and the coefficients of the polarization vector, $\mathbf{p}=\mathbf{u} /|\mathbf{u}|$, are with respect to the global specimen Cartesian basis. Of the impacts considered in this work, only impact on (100) results in propagation of a pure longitudinal wave. In all cases, the quasi- or pure-longitudinal wave has a wave speed that is approximately twice that for quasi- or pure-shear waves. In the case of impact on (210), the arrival time of the quasi-shear wave is sufficiently late that it cannot account for the delayed slip activity observed in Fig. 9.

The delayed activation of secondary slip systems might also be explained by reorientation of slip systems or stress due to slip activity on primary systems. Consider the slip-system resolved shear stress expressed as $\tau^{\alpha}=\mathbf{s}^{\alpha} \cdot \boldsymbol{\sigma} \cdot \boldsymbol{n}^{\alpha}$. An eigenvalue decomposition of the Cauchy stress into $\boldsymbol{\sigma}=\sum_{k} \lambda_{k} \mathbf{v}_{k} \otimes \mathbf{v}_{k}$ enables the resolved shear stress to be expressed in terms of the alignment between eigenvectors of the applied loading and the slip system geometry, i.e.

$$
\tau^{\alpha}=\sum_{k=1}^{3} \lambda_{k}\left(\mathbf{s}^{\alpha} \cdot \mathbf{v}_{k}\right)\left(\mathbf{v}_{k} \cdot \boldsymbol{n}^{\alpha}\right)
$$

From this expression it is evident that, under loading with one non-zero eigenvalue, maximal values of resolved shear stress occur for slip systems favorably oriented such that the normal and slip direction are aligned $45^{\circ}$ away from the eigenvector, thus leading to the classical definition of Schmid factor for uniaxial stress states. For the more general case, there are contributions from each eigenvalue weighed by the alignment of the corresponding eigenvector with the slip system geometry. Using this expression, we examine the potential 
Table 7: Estimated infinitesimal elastic wave speeds and polarization vector for impacts on single crystal RDX with orientations considered in this work.

\begin{tabular}{llrrrll}
\hline & & wave speed & \multicolumn{3}{c}{ polarization vector } & \\
& id & $(\mathrm{m} / \mathrm{s})$ & $p_{1}$ & $p_{2}$ & $p_{3}$ & description \\
\hline Impact on (100): & & & & & & \\
& 1 & 3725.3 & 1.000 & 0.000 & 0.000 & pure longitudinal \\
& 2 & 1957.9 & 0.000 & 1.000 & 0.000 & pure shear \\
& 3 & 1501.9 & 0.000 & 0.000 & 1.000 & pure shear \\
Impact on (210): & & & & & & \\
& 1 & 3624.0 & 0.997 & -0.073 & 0.000 & quasi longitudinal \\
& 2 & 1960.3 & 0.073 & 0.997 & 0.000 & quasi shear \\
& 3 & 1550.4 & 0.000 & 0.000 & 1.000 & pure shear \\
Impact on (021): & & & & & & \\
& 1 & 3195.6 & 0.997 & 0.000 & -0.075 & quasi longitudinal \\
& 2 & 1820.6 & 0.075 & -0.000 & 0.997 & quasi shear \\
& 3 & 1864.6 & 0.000 & 1.000 & 0.000 & pure shear \\
Impact on (111): & & & & & & \\
& 1 & 3180.5 & 0.992 & -0.028 & -0.126 & quasi longitudinal \\
& 2 & 1975.4 & -0.123 & -0.487 & -0.864 & quasi shear \\
& 3 & 1783.9 & 0.037 & -0.873 & 0.487 & quasi shear \\
\hline
\end{tabular}

contributors to the delayed activation of secondary slip systems in (210) impact. Firstly, a reorientation of $\mathbf{s}^{\alpha}$ and $\mathbf{n}^{\alpha}$ by $\mathbf{F}_{\mathrm{e}}$ or reorientation of the stress direction, $\mathbf{v}_{k}$, as plasticity evolves could promote slip on additional slip systems. Alternately, for fixed orientations of $\mathbf{s}^{\alpha}, \mathbf{n}^{\alpha}$, and $\mathbf{v}_{k}$ during loading, it is possible that the evolution of $\lambda_{k}$ can also shift the slip activity from one set of slip systems to another.

The plot in Fig. 10 depicts the pole normal to each of the relevant slip planes (for shot 1302) and the corresponding slip direction vectors. In this plot, red and blue markers indicate slip plane normal and slip directions, respectively, while black markers indicate specimen axes. The (210) pole is aligned with the impact direction, X, consistent with shot 1302. Distinct marker shapes are used for each slip system, e.g. blue and red circles correspond to slip system 5, (010)[100], and are labeled by ID number consistent with Table 1. Repeated symbols correspond to the same vector computed at three different times in correspondence with the red circle markers in Fig. 9a, viz. prior to the arrival of the elastic wave, at the peak in $\tau_{5}$, and finally at the peak in $\tau_{12}$ and $\tau_{13}$. There is no appreciable reorientation of the slip system geometry; the largest reorientation angle is less than $2^{\circ}$ as apparent from Fig. 10 by the nearly identically overlapping symbols representing different times in deformation process. The purple markers on this plot indicate the eigenvectors of the Cauchy stress at two times. There is a slight reorientation of the dominant stress axes about the Z-axis by approximately $2.7^{\circ}$.

In this case, we conclude that the cause of delayed activation of secondary slip systems observed in Figs. 8 and 9 is that the rate of plastic deformation associated with slip on (010)[100] and (110)[110] (IDs 5 and 10 , respectively) is not coaxial with the Cauchy stress. Consequently, the decrease in projected shear stress on those systems is accommodated by inelastic strains which generate an increase in the components of elastic strain and stress more closely aligned with $\mathbf{v}_{3}$. In other words, while the eigenvectors of the stress state do not significantly reorient, there is a significant reduction and increase in the eigenvalues associated with $\mathbf{v}_{2}$ and $\mathbf{v}_{3}$, respectively, as listed in Table 8. This shift of stress toward $\mathbf{v}_{3}$ eventually favors slip on $\alpha=12,13$ over $\alpha=5,10$. This behavior is a consequence of the low symmetry of the material that would not otherwise be expected; in higher symmetry crystals, there are sufficient slip systems such that plastic deformation is approximately co-axial with the applied stress regardless of loading direction. Quasistatic deformation studies of RDX have also exhibited this same effect (cf. Ramos et al., 2009, Fig 6.).

Figures 11a and 11b illustrate the spatial distribution of mobile dislocation density across the shock wave 
Table 8: Shift in eigenvalues of deviatoric part of Cauchy stress during passage of shock wave for shot 1302.

\begin{tabular}{rrrr}
\hline Distance $(\mathrm{mm})$ from wavefront (cf. Fig 9$)$ & $\lambda_{1}(\mathrm{MPa})$ & $\lambda_{2}(\mathrm{MPa})$ & $\lambda_{3}(\mathrm{MPa})$ \\
\hline+0.050 & 0.000 & 0.000 & 0.000 \\
-0.025 & -306.9 & 117.1 & 189.8 \\
-0.125 & -319.1 & 34.31 & 284.8 \\
\hline
\end{tabular}

for shot 1354 (1.4 GPa) and 1302 (2.9 GPa), respectively. For the lower pressure shot (i.e. 1354), dislocations evolve most rapidly on $\{021\}$ [100], but saturate to approximately $5 \times 10^{10} \mathrm{~cm}^{-2}$, while dislocations on other active systems continue to evolve and accommodate a significant portion of the plastic deformation. Note, for the model parameters used in these simulations, there is no explicit saturation value of dislocation density, i.e. $C_{\mathrm{A}}=0$. The lag between activity on $\{011\}\langle 01 \overline{1}\rangle$ behind that for $(010)[100]$ plus $(110)[1 \overline{1} 0]$ is consistent with Figures 8 and 9.

Figure 12 depicts the spatial distribution of accumulated plastic slip on systems active for impact along (111) orientation from simulations of (a) shot 1357 (1.1 GPa) and (b) shot 1311 (2.9 GPa), respectively. The transition from a well-separated two wave structure at the lower shock pressure to the overdriven single shock wave observed in Figure 6 is also evident by the simultaneous slip system activity exhibited near the shock front in Figure 12b for the higher pressure case, but not in Figure 12a for the lower pressure case where there is clear separation amongst slip activity on different systems $0.2 \mathrm{~mm}$ behind the shock front.

\section{Summary and Conclusions}

We have developed a dislocation density-based model for the anisotropic response of RDX crystals under shock loading conditions that accounts for nonlinear thermoelastic lattice deformation through a free energybased EOS (Cawkwell et al., 2016) in combination with temperature and pressure dependent elastic constants, as well as crystallographic slip associated with dislocation motion on several distinct slip systems. The six slip systems used in earlier models of $\alpha$-RDX are unable to completely account for plastic deformation inferred from interface velocimetry of plate impact experiments; therefore, we have included seven new slip systems, comprising three families, based on Burgers vectors observed in X-ray topography by Halfpenny et al. (1986). These new slip systems evidently provide a good description of the anisotropic elastic-plastic response of RDX. While we tried to further reduce this set of slip systems, the agreement between model and experiment was degraded for every subset of the full thirteen systems that was considered. Further experiments including indentation, X-ray topography, and in situ X-ray diffraction are necessary to assess the validity of the set of slip systems proposed here.

The evolution kinetics of plastic deformation incorporate the expression for dislocation velocity developed by Austin and McDowell (2011), which accounts for the transition from thermally-activated to drag-limited regimes of dislocation motion, and dislocation evolution behavior including generation from the interaction of mobile dislocations with forest obstacles, annihilation amongst co-planar dislocation segments of opposite polarity, and the demobilization of dislocations as they encounter forest obstacles. Many of the model parameters have a physical basis and, accordingly, their values are determined from physical properties of RDX observed experimentally or quantified from atomistic calculations. The remaining model parameters were calibrated by fitting to velocimetry measurements from a single experiment corresponding to a $2.9 \mathrm{GPa}$ shock wave normal to the (210) planes of an approximately $1.5 \mathrm{~mm}$ thick target specimen of $\alpha$-RDX. After calibrating the model to data from this single experiment, the same model parameters were used to simulate a total of 29 experiments comprising four crystallographic orientations, a range of shock pressures, varying anvil and window materials, and several specimen thicknesses. The simulations produced results in strong agreement with the experimental data considering both the observed experimental variability and the range of experimental configurations.

There are several conclusions we make based on this work. Firstly, the proposed slip systems and dislocation-density based slip kinetics are sufficient to capture features of velocimetry consistent with plas- 


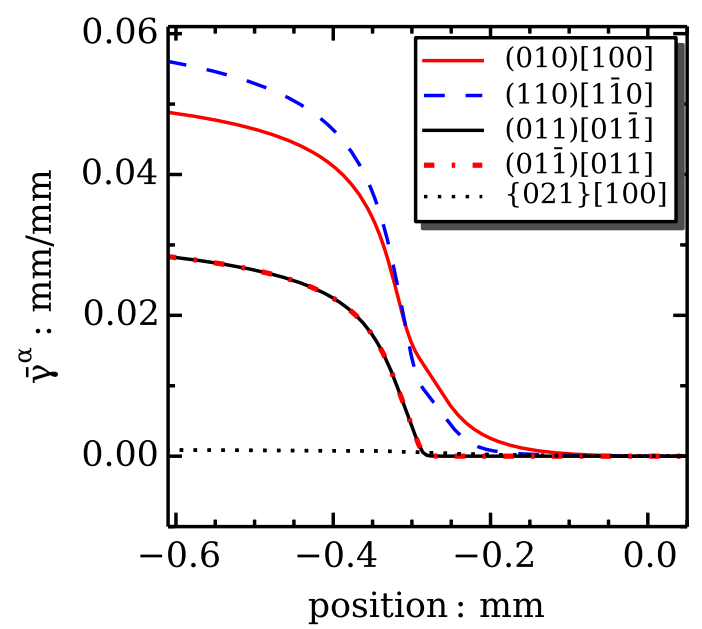

(a) shock pressure $\approx 1.3 \mathrm{GPa}$

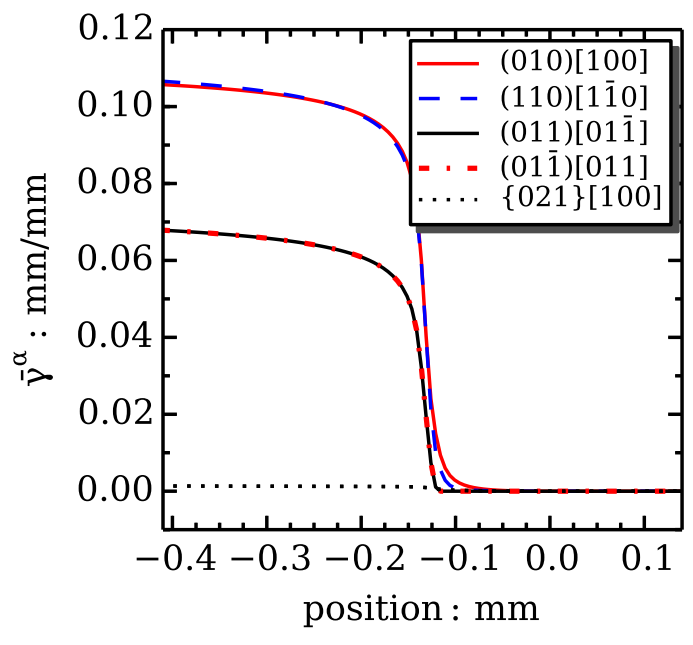

(b) shock pressure $\approx 3.0 \mathrm{GPa}$

Figure 8: Spatial profiles of accumulated slip on slip systems for impact along (210) for (a) lower and (b) higher pressure shots. Position is with respect to shock front (i.e. $x=0$ ). Significant accumulation of slip develops approximately $0.2 \mathrm{~mm}$ and $0.1 \mathrm{~mm}$ behind the shock front for the $\approx 1.3 \mathrm{GPa}$ and $\approx 3 \mathrm{GPa}$ shocks, respectively.

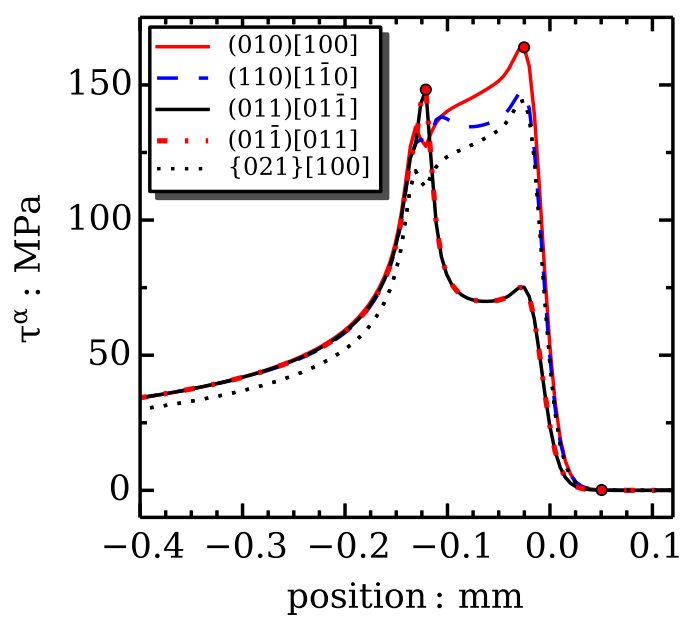

(a)

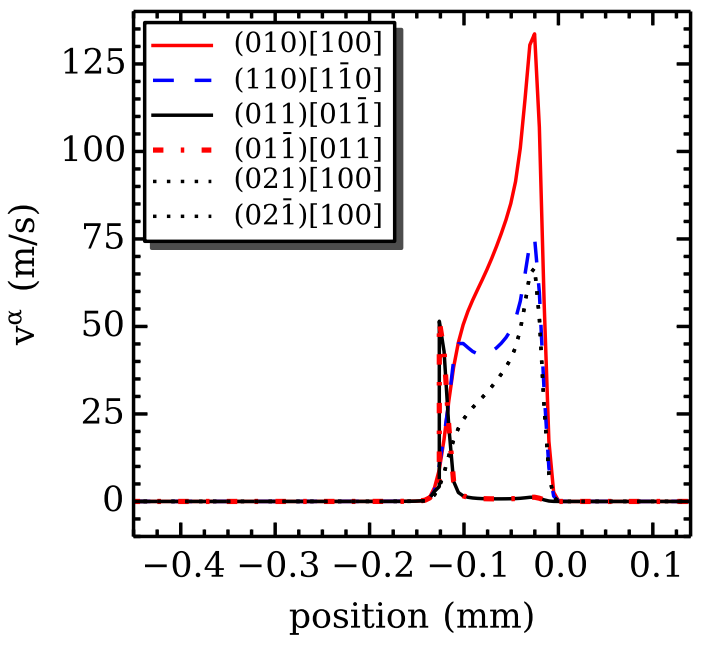

(b)

Figure 9: Spatial profiles of (a) projected shear stress on each slip system and (b) the corresponding dislocation velocity as shock wave progresses through specimen for shot 1302. Position is with respect to shock front (i.e. $x=0$ ). There is an apparent delay in dislocation activity on $\{01 \overline{1}\}<011>$ attributed to temporary relaxation and subsequent reloading on that plane and in correspondence with delayed accumulation of slip evident in Fig. 8. 


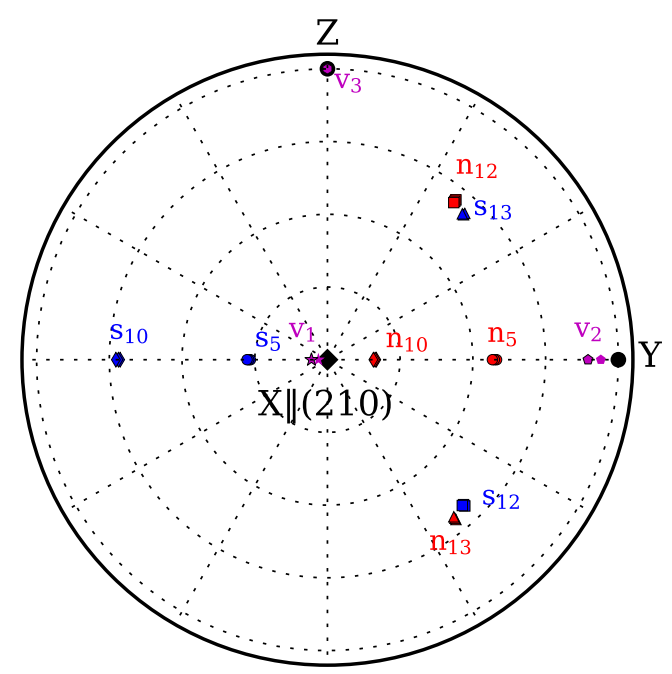

Figure 10: Pole figure illustrating that only negligible re-orientation of slip planes or stress occur during the passage of shock wave along (210). Red and blue symbols indicate slip plane normal and slip direction, respectively, while purple markers indicate eigenvectors of Cauchy stress. The figure is oriented such that the shock direction is at the center.

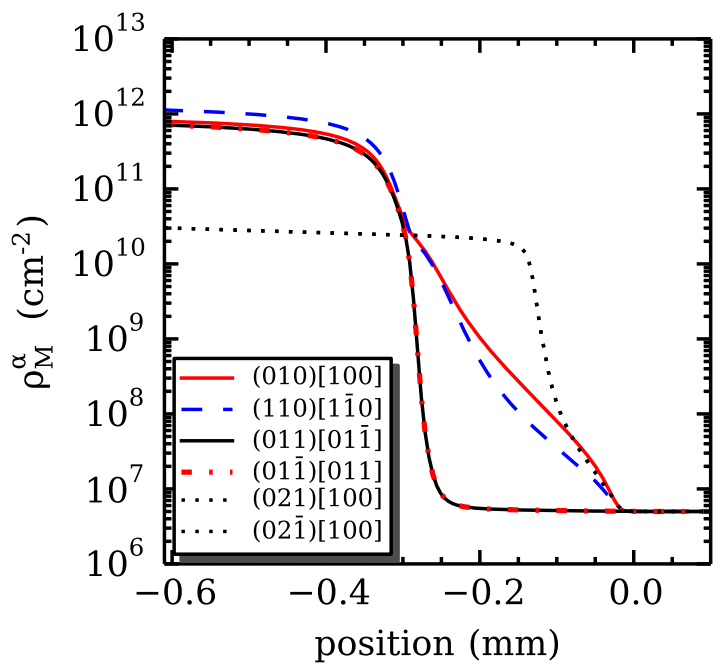

(a) shock pressure $\approx 1.3 \mathrm{GPa}$

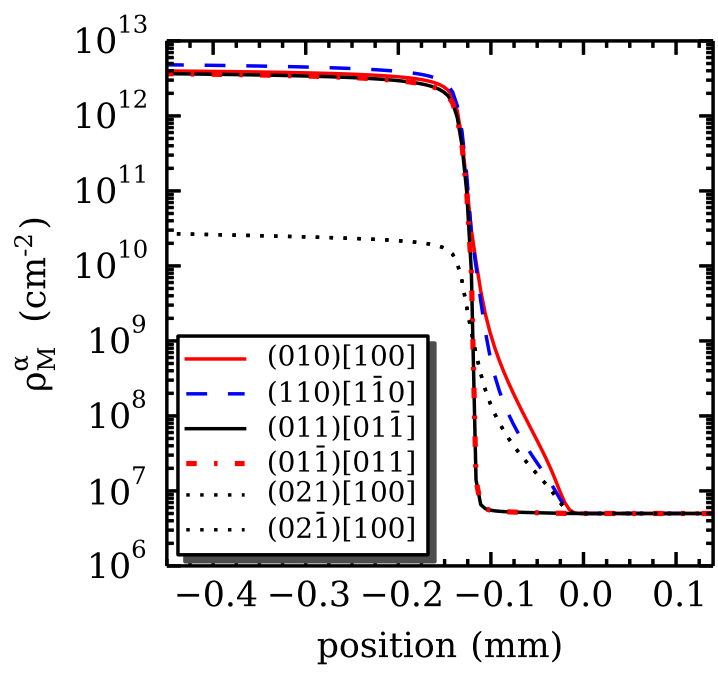

(b) shock pressure $\approx 3.0 \mathrm{GPa}$

Figure 11: Spatial profiles of dislocation density with respect to shock front for impact along (210). For the lower pressure shock (a) dislocations on $\{021\}[100]$ initially evolve at a higher rate than other slip systems, although this evolution is suppressed and the associated slip activity on the system is negligible in comparison with other systems. 


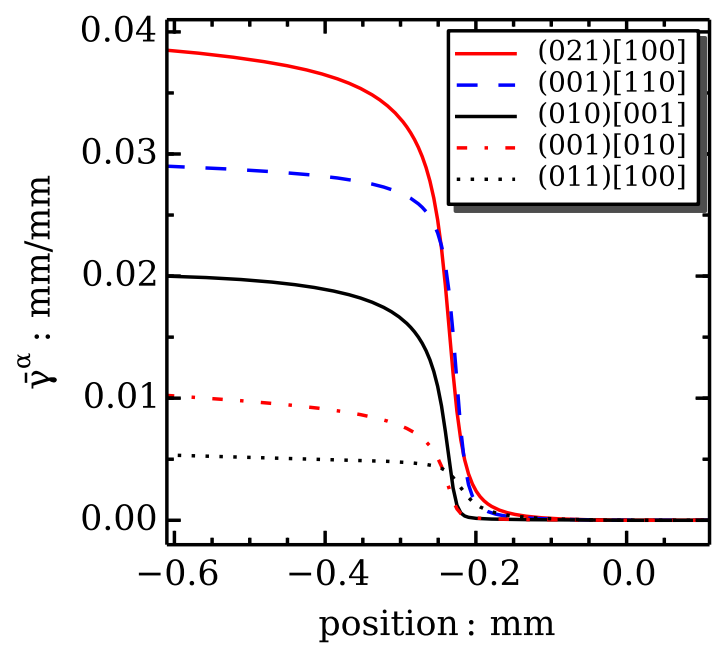

(a) shock pressure $\approx 1.3 \mathrm{GPa}$

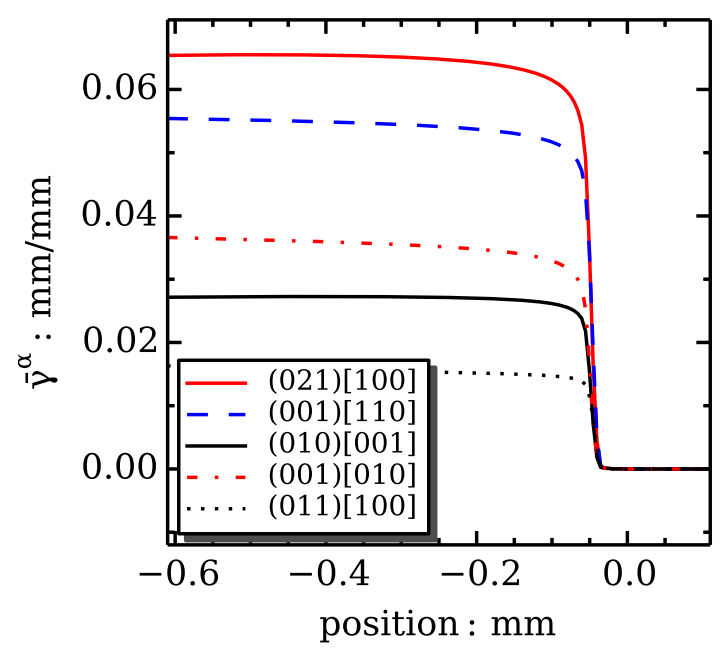

(b) shock pressure $\approx 3.0 \mathrm{GPa}$

Figure 12: Spatial profiles of accumulated slip on slip systems for impact along (111) for (a) lower and (b) higher pressure shots. Position is with respect to shock front (i.e. $x=0$ ). Significant accumulation of slip develops approximately $0.2 \mathrm{~mm}$ behind the shock front for the (a) lower (i.e. $\approx 1.3 \mathrm{GPa}$ ) pressure shock; whereas, the overdriven nature of the (b) $3.0 \mathrm{GPa}$ shock wave is evident from the near coincidence of wave front and plastic deformation.

ticity of RDX in several crystallographic orientations. Also, because of the low initial dislocation density in these crystals, rapid dislocation generation is critical to accommodate impact deformation through plasticity. The low initial dislocation density and rapid generation of dislocations needed in the model results in a transition from drag-limited dislocation velocity in the immediate wake of the elastic precursor to a slower thermally-activated regime throughout the plastic wave front.

The model is applicable to simulating the response of RDX in shock-loading or impact environments such that the RDX remains in its ambient $\alpha$ polymorph. For conditions including solid-solid phase transformations (e.g. $\alpha$ to $\gamma$ or $\epsilon$ polymorphs) or melting, kinematics and kinetics of phase transformation such as that developed by Addessio et al. (2016) or Barton et al. (2009), respectively, must be added to the model developed here. As discussed in Section 4, the loading conditions examined in this paper are clearly below the known shock pressures and temperatures required for phase transformations, including melt. We envision application of this model to simulating the evolution of heterogeneous plasticity and temperature fields in polycrystalline explosive materials caused by weak shock events.

\section{Acknowledgments}

This work was performed under the auspices of the U.S. Department of Energy under contract DE-AC5206NA25396. In particular, the authors are grateful for the support of the Laboratory Directed Research and Development (LDRD) program's Exploratory Research project (ER20140643) targeting Materials in Extreme Environments. The authors are grateful for discussions of dislocation kinetics and interactions with J. Mayeur.

\section{References cited}

Addessio, F., Luscher, D., Cawkwell, M., Ramos, K., 2016. A high-rate, single-crystal model for RDX including phase transformation and plastic slip. in preparation.

Alankar, A., Eisenlohr, P., Raabe, D., 2011. A dislocation density-based crystal plasticity constitutive model for prismatic slip in $\alpha$-titanium. Acta Materialia 59 (18), 7003-7009. 
Alleman, C. N., Ghosh, S., Luscher, D. J., Bronkhorst, C. A., 2014. Evaluating the effects of loading parameters on single-crystal slip in tantalum using molecular mechanics. Philosophical Magazine 94 (1), $92-116$.

Amelinckx, S., 1956. The direct observation of dislocation nets in rock salt single crystals. Philosophical Magazine 1 (3), 269-290.

Asaro, R. J., 1983. Crystal plasticity. Journal of Applied Mechanics 50 (4b), 921-934.

Austin, R. A., Barton, N. R., Howard, W. M., Fried, L. E., 2014. Modeling pore collapse and chemical reactions in shock-loaded HMX crystals. In: Journal of Physics: Conference Series. Vol. 500. IOP Publishing, p. 052002 .

Austin, R. A., Barton, N. R., Reaugh, J. E., Fried, L. E., 2015. Direct numerical simulation of shear localization and decomposition reactions in shock-loaded HMX crystal. Journal of Applied Physics 117 (18), 185902.

Austin, R. A., McDowell, D. L., Jan. 2011. A dislocation-based constitutive model for viscoplastic deformation of fcc metals at very high strain rates. International Journal of Plasticity 27 (1), 1-24.

Barton, N. R., Winter, N. W., Reaugh, J. E., 2009. Defect evolution and pore collapse in crystalline energetic materials. Modelling and Simulation in Materials Science and Engineering 17 (3), 035003.

Bolme, C. A., Ramos, K. J., 2014. The elastic tensor of single crystal RDX determined by Brillouin spectroscopy. Journal of Applied Physics 116 (18), 183503.

Bowden, F. P., Yoffe, A., 1948. Hot spots and the initiation of explosion. In: Symposium on Combustion and Flame, and Explosion Phenomena. Vol. 3. Elsevier, pp. 551-560.

Bowden, F. P., Yoffe, A. D., 1952. Initiation and growth of explosion in liquids and solids. Cambridge University Press.

Bronkhorst, C. A., Kalidindi, S. R., Anand, L., 1992. Polycrystalline plasticity and the evolution of crystallographic texture in FCC metals. Philosophical Transactions of the Royal Society of London. Series A: Physical and Engineering Sciences 341 (1662), 443-477.

Cady, H. H., 1972. Coefficient of thermal expansion of pentaerythritol tetranitrate and hexahydro-1, 3, 5-trinitro-s-triazine (RDX). Journal of Chemical and Engineering Data 17 (3), 369-371.

Cawkwell, M. J., Luscher, D. J., Addessio, F. L., Ramos, K. J., 2016. Equations of state for the $\alpha$ and $\gamma$ polymorphs of cyclotrimethylene trinitramine. Journal of Applied Physics 119 (18), 185106.

Cawkwell, M. J., Ramos, K. J., Hooks, D. E., Sewell, T. D., 2010. Homogeneous dislocation nucleation in cyclotrimethylene trinitramine under shock loading. Journal of Applied Physics 107 (6), 063512.

Cawkwell, M. J., Sewell, T. D., Zheng, L., Thompson, D. L., 2008. Shock-induced shear bands in an energetic molecular crystal: Application of shock-front absorbing boundary conditions to molecular dynamics simulations. Physical Review B 78 (1), 014107.

Choi, C. S., Prince, E., 1972. The crystal structure of cyclotrimethylenetrinitramine. Acta Crystallographica Section B: Structural Crystallography and Crystal Chemistry 28 (9), 2857-2862.

Clayton, J. D., 2005. Dynamic plasticity and fracture in high density polycrystals: constitutive modeling and numerical simulation. Journal of the Mechanics and Physics of Solids 53 (2), 261-301.

Clayton, J. D., 2011. Nonlinear Mechanics of Crystals. Vol. 177 of Solid Mechanics and Its Applications. Springer Verlag. 
Clayton, J. D., Becker, R., 2012. Elastic-plastic behavior of cyclotrimethylene trinitramine single crystals under spherical indentation: Modeling and simulation. Journal of Applied Physics 111 (6), 063512.

De, S., Zamiri, A. R., et al., 2014. A fully anisotropic single crystal model for high strain rate loading conditions with an application to $\alpha$-RDX. Journal of the Mechanics and Physics of Solids 64, 287-301.

Devincre, B., Kubin, L., Hoc, T., 2006. Physical analyses of crystal plasticity by DD simulations. Scripta Materialia 54 (5), 741-746.

Dreger, Z. A., Gupta, Y. M., 2010. Phase diagram of hexahydro-1, 3, 5-trinitro-1, 3, 5-triazine crystals at high pressures and temperatures. The Journal of Physical Chemistry A 114 (31), 8099-8105.

Elban, W. L., Armstrong, R. W., 1982. Microhardness study of RDX to assess localized deformation and its role in hot spot formation. In: Proceedings of the Seventh Symposium (International) on Detonation. Naval Surface Weapons Center Silver Spring, Maryland.

Elban, W. L., Armstrong, R. W., Russell, T. P., 1998. Plasticity/interfacial energy influences on combustiondriven cracking of rdx energetic crystals. Philosophical Magazine A 78 (4), 907-912.

Elban, W. L., Armstrong, R. W., Yoo, K. C., Rosemeier, R. G., Yee, R. Y., 1989. X-ray reflection topographic study of growth defect and microindentation strain fields in an RDX explosive crystal. Journal of materials science 24 (4), 1273-1280.

Elban, W. L., Hoffsommer, J. C., Armstrong, R. W., 1984. X-ray orientation and hardness experiments on RDX explosive crystals. Journal of materials science 19 (2), 552-566.

Gallagher, H. G., Halfpenny, P. J., Miller, J. C., Sherwood, J. N., Tabor, D., 1992. Dislocation slip systems in pentaerythritol tetranitrate (PETN) and cyclotrimethylene trinitramine (RDX). Philosophical Transactions of the Royal Society of London A: Mathematical, Physical and Engineering Sciences 339 (1654), 293-303.

Gibbs, T. R., 1980. LASL explosive property data. Vol. 4. Univ of California Press.

Halfpenny, P. J., Roberts, K. J., Sherwood, J. N., 1984. Dislocations in energetic materials: Part 3. Etching and microhardness studies of pentaerythritol tetranitrate and cyclotrimethylenetrintitramine. Journal of Materials Science 19, 1629.

Halfpenny, P. J., Roberts, K. J., Sherwood, J. N., 1986. Dislocations in energetic materials dislocation characterization and post-growth motion in single crystals of cyclotrimethylene trinitramine. Philosophical Magazine A 53 (4), 531-542.

Haussühl, S., 2001. Elastic and thermoelastic properties of selected organic crystals. Zeitschrift für Kristallographie/International journal for structural, physical, and chemical aspects of crystalline materials $216(6 / 2001), 339-353$.

Hooks, D. E., Ramos, K. J., Bolme, C. A., Cawkwell, M. J., 2015. Elasticity of crystalline molecular explosives. Propellants, Explosives, Pyrotechnics 40 (3), 333-350.

Hooks, D. E., Ramos, K. J., Martinez, A. R., 2006. Elastic-plastic shock wave profiles in oriented single crystals of cyclotrimethylene trinitramine (RDX) at 2.25 GPa. Journal of applied physics 100 (2), 024908.

Hooks, D. E., Ramos, K. J., Martinez, A. R., 2011. Erratum:"Elastic-plastic shock wave profiles in oriented single crystals of cyclotrimethylene trinitramine (RDX) at $2.25 \mathrm{GPa}$ "[j. appl. phys. 100, 024908 (2006)]. Journal of Applied Physics 109 (8), 089901.

Josyula, K., Rahul, De, S., 2016. Quasi-static response and texture evolution of $\alpha$-and $\gamma$-RDX: a comparative study. Philosophical Magazine, 1-19. 
Kalidindi, S., Bronkhorst, C., Anand, L., 1992. Crystallographic texture evolution in bulk deformation processing of FCC metals. Journal of the Mechanics and Physics of Solids 40 (3), 537-569.

Kocks, U. F., Argon, A. S., Ashby, M. F., 1975. Thermodynamics and kinetics of slip. Progress In Materials Science 19, 1-281.

Kubin, L., Devincre, B., Hoc, T., 2008. Modeling dislocation storage rates and mean free paths in facecentered cubic crystals. Acta materialia 56 (20), 6040-6049.

Lee, M. G., Lim, H., Adams, B. L., Hirth, J. P., Wagoner, R. H., 2010. A dislocation density-based single crystal constitutive equation. International Journal of Plasticity 26 (7), 925-938.

Lloyd, J. T., Clayton, J. D., Austin, R. A., McDowell, D. L., Sep. 2014. Plane wave simulation of elasticviscoplastic single crystals. Journal of the Mechanics and Physics of Solids 69, 14-32.

Luscher, D. J., Bronkhorst, C. A., Alleman, C. N., Addessio, F. L., Sep. 2013. A model for finite-deformation nonlinear thermomechanical response of single crystal copper under shock conditions. Journal of the Mechanics and Physics of Solids 61 (9), 1877-1894.

Luscher, D. J., Mayeur, J. R., Mourad, H. M., Hunter, A., Kenamond, M. A., 2016. Coupling continuum dislocation transport with crystal plasticity for application to shock loading conditions. International Journal of Plasticity 76, 111-129.

Ma, A., Roters, F., 2004. A constitutive model for fcc single crystals based on dislocation densities and its application to uniaxial compression of aluminium single crystals. Acta materialia 52 (12), 3603-3612.

Ma, A., Roters, F., Raabe, D., 2006. A dislocation density based constitutive model for crystal plasticity FEM including geometrically necessary dislocations. Acta Materialia 54 (8), 2169-2179.

Mathur, K. K., Dawson, P. R., 1989. On modeling the development of crystallographic texture in bulk forming processes. International Journal of Plasticity 5 (1), 67-94.

Mayeur, J. R., Mourad, H. M., Luscher, D. J., Hunter, A., Kenamond, M. A., 2016. Numerical implementation of a crystal plasticity model with dislocation transport for high strain rate applications. Modelling and Simulation in Materials Science and Engineering 24 (4), 045013.

McDermott, I. T., Phakey, P. P., 1971. An x-ray topographic study of defect structures in cyclotrimethylene trinitramine. Physica Status Solidi (A) 8 (2), 505-511.

Prasad, G., Goerdeler, M., Gottstein, G., 2005. Work hardening model based on multiple dislocation densities. Materials Science and Engineering: A 400, 231-233.

Ramos, K. J., Bahr, D. F., Hooks, D. E., 2011. Defect and surface asperity dependent yield during contact loading of an organic molecular single crystal. Philosophical Magazine 91 (7-9), 1276-1285.

Ramos, K. J., Cawkwell, M. J., Bolme, C. A., Hooks, D. E., 2014a. Effects of orientation, pressure/strain rate, and microstructure on the quasi-static deformation and shock response of single crystal explosives. In: of Naval Research, O. (Ed.), Proceedings of the 15th International Detonation Symposium.

Ramos, K. J., Hooks, D. E., Bahr, D. F., 2009. Direct observation of plasticity and quantitative hardness measurements in single crystal cyclotrimethylene trinitramine by nanoindentation. Philosophical Magazine 89 (27), 2381-2402.

Ramos, K. J., Hooks, D. E., Sewell, T. D., Cawkwell, M. J., 2010. Anomalous hardening under shock compression in (021)-oriented cyclotrimethylene trinitramine single crystals. Journal of Applied Physics $108(6), 066105$. 
Ramos, K. J., Hooks, D. E., et al., 2016. Elastic-plastic shock wave profiles in oriented single crystals of cyclotrimethylene trinitramine $(\mathrm{RDX})$ at $1.3 \mathrm{GPa}$. in preparation.

Ramos, K. J., Jensen, B. J., Iverson, A. J., Yeager, J. D., Carlson, C. A., Montgomery, D. S., Thompson, D. G., Fezzaa, K., Hooks, D. E., 2014b. In situ investigation of the dynamic response of energetic materials using IMPULSE at the Advanced Photon Source. In: Journal of Physics: Conference Series. Vol. 500. IOP Publishing, p. 142028.

Roters, F., Raabe, D., Gottstein, G., 2000. Work hardening in heterogeneous alloys - a microstructural approach based on three internal state variables. Acta Materialia 48 (17), 4181-4189.

Scheidler, M., Wright, T. W., 2001. A continuum framework for finite viscoplasticity. International Journal of Plasticity 17 (8), 1033-1085.

Schuler, K. W., 1970. Propagation of steady shock waves in polymethyl methacrylate. Journal of the Mechanics and Physics of Solids 18 (4), 277-293.

Schuler, K. W., Nunziato, J. W., 1974. The dynamic mechanical behavior of polymethyl methacrylate. Rheologica Acta 13 (2), 265-273.

Sewell, T. D., Bennett, C. M., 2000. Monte carlo calculations of the elastic moduli and pressure-volumetemperature equation of state for hexahydro-1, 3, 5-trinitro-1, 3, 5-triazine. Journal of Applied Physics 88 (1), 88-95.

Sharma, J., Armstrong, R. W., Elban, W. L., Coffey, C. S., Sandusky, H. W., 2001. Nanofractography of shocked rdx explosive crystals with atomic force microscopy. Applied Physics Letters 78 (4), 457-459.

Steinberg, D., et al., 1996. Equation of state and strength properties of selected materials. Lawrence Livermore National Laboratory Livermore, CA.

Taylor, D. E., 2014. Pressure dependent elastic constants of alpha and gamma cyclotrimethylene trinitramine: A quantum mechanical study. Journal of Applied Physics 116 (5), 053513.

Wiegand, D. A., Pinto, J., Nicolaides, S., 1991. The mechanical response of tnt and a composite, composition b, of tnt and rdx to compressive stress: I uniaxial stress and fracture. Journal of energetic materials 9 (1-2), $19-80$.

Winey, J. M., Gupta, Y. M., 2006. Nonlinear anisotropic description for the thermomechanical response of shocked single crystals: Inelastic deformation. Journal of Applied Physics 99 (2), 023510.

Winey, J. M., Gupta, Y. M., 2010. Anisotropic material model and wave propagation simulations for shocked pentaerythritol tetranitrate single crystals. Journal of Applied Physics 107 (10), 103505.

Wood, M. A., Cherukara, M. J., Kober, E. M., Strachan, A., 2015. Ultrafast chemistry under nonequilibrium conditions and the shock to deflagration transition at the nanoscale. The Journal of Physical Chemistry C 119 (38), 22008-22015.

Ziegler, H., 1983. An introduction to thermomechanics. North-Holland series in applied mathematics and mechanics. North-Holland Pub. Co. 